\title{
Article \\ A Novel Approach of Unit Conversion in the Lattice Boltzmann Method
}

\author{
Saleh S. Baakeem ${ }^{1, *}$, Saleh A. Bawazeer ${ }^{2}$ and Abdulmajeed. A. Mohamad ${ }^{1}$ (D) \\ 1 Department of Mechanical and Manufacturing Engineering, Schulich School of Engineering, \\ University of Calgary, Calgary, AB T2N 1N4, Canada; mohamad@ucalgary.ca \\ 2 Mechanical Engineering Department, College of Engineering and Islamic Architecture, \\ Umm Al-Qura University, P.O. 5555, Makkah 24382, Saudi Arabia; sambawazeer@uqu.edu.sa \\ * Correspondence: saleh.baakeem@ucalgary.ca
}

Citation: Baakeem, S.S.; Bawazeer

S.A.; Mohamad, A..A. A Novel

Approach of Unit Conversion in the Lattice Boltzmann Method. Appl. Sci. 2021, 11, 6386. https://doi.org/ 10.3390/app11146386

Academic Editors: Tomonobu Senjyu, Sébastien Poncet and Seyed Soheil Mousavi Ajarostaghi

Received: 10 June 2021

Accepted: 8 July 2021

Published: 10 July 2021

Publisher's Note: MDPI stays neutral with regard to jurisdictional claims in published maps and institutional affiliations.

Copyright: (c) 2021 by the authors. Licensee MDPI, Basel, Switzerland. This article is an open access article distributed under the terms and conditions of the Creative Commons Attribution (CC BY) license (https:// creativecommons.org/licenses/by/ $4.0 /)$.

\begin{abstract}
The lattice Boltzmann method (LBM) is an alternative method to the conventional computational fluid dynamic (CFD) methods. It gained popularity due to its simplicity in coding and dealing with a complex fluid flow such as the multiphase flow. The method is based on the kinetic theory, which is mesoscopic scale. Hence, applying the LBM method for macroscopic problems requires a proper conversion from the physical scale (conventional units) to the mesoscopic scale (lattice units) and vice versa. The Buckingham $\pi$ theorem and the principle of corresponding states are the popular methods used for data reductions and unit conversion processes in the LBM. Nevertheless, those methods have some issues, such as difficulty in converting specific quantities, such as thermophysical properties. The current work uses a novel dimensional analysis method systematically for mapping properties' units between scales. Moreover, the approach has the flexibility in selecting parameters to ensure the stability of the method of solution. Several benchmark examples are used to evaluate the feasibility and accuracy of the proposed approach. In conclusion, the proposed approach showed the flexibility of the mapping between meso-scale to macro-scales and vice versa on solid bases rather than ad-hoc methods.
\end{abstract}

Keywords: lattice Boltzmann method; unit conversion; advection-diffusion; phase change; differentially heated cavity; surface tension

\section{Introduction}

The problems encountered in engineering and sciences are either macroscopic, mesoscopic, microscopic in nature, or a combination of them, see Figure 1. At the macroscopic level, the problems are a continuum. Whereas, at the microscopic level, the material is treated as soft or solid particles. The lattice Boltzmann method (LBM) is based on meso-scales. The LBM became a very popular method since its birthday in late 1989. One of the main advantages of the LBM is its ability to incorporate the microscopic or mesoscopic physics while recovering the macroscopic laws at an affordable computational cost [1-4]. Therefore, converting or mapping quantities' units from/to the macroscopic scale (physical-scale) to/from the mesoscopic scale (lattice-scale) should correctly be performed. The geometric and dynamics similarity conditions must be conserved between the physical-scale and lattice-scale. The most popular method is to match the dimensionless parameters (such as Reynolds number, Rayleigh number, etc.) besides the geometrical similarity, such as aspect ratio, i.e., the Buckingham $\pi$ theorem. For single-phase and single component flows and heat and mass transfer, the controlling dimensionless parameters are few and easy to match. However, the problem of using similarity transformation for multi-phase flows and transports is not straightforward. In addition, the same argument equally applies to problems with variable thermo-physical properties and non-Newtonian flow. For completeness of the topic, a short introduction will be discussed on the current 
methods used for mapping processes from the physical domain to the lattice domain and vice versa, especially for multi-phase problems.

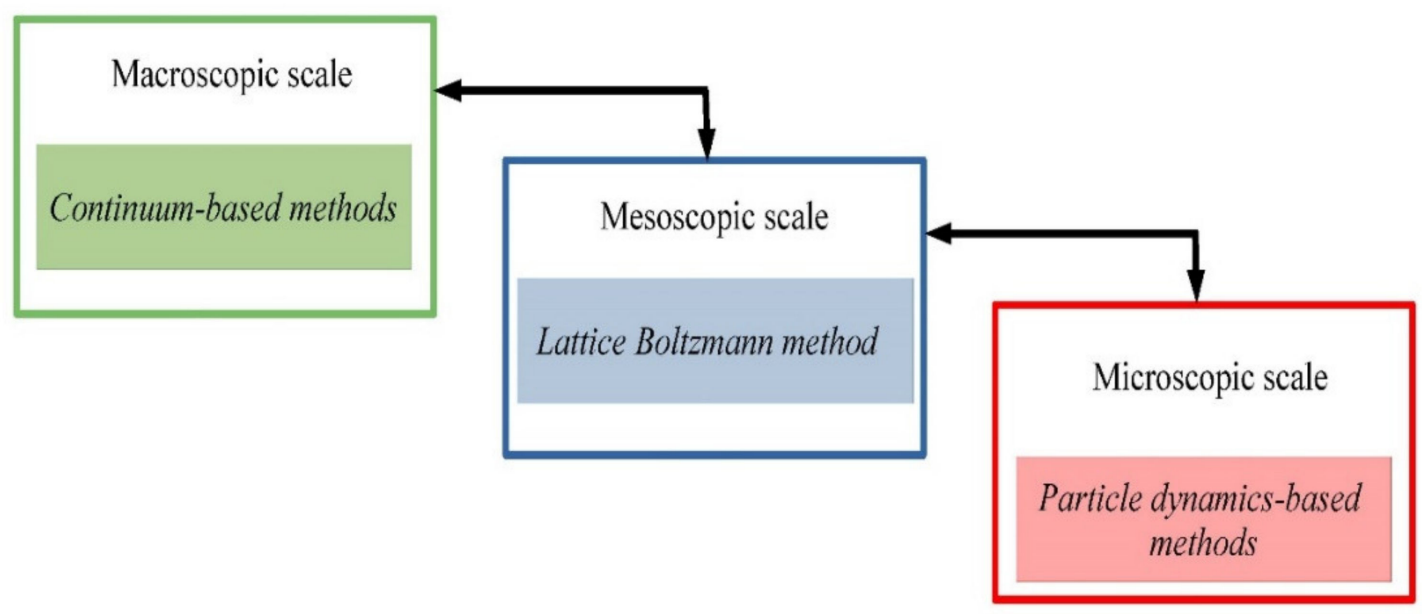

Figure 1. A hierarchy of scales and simulation levels.

The Buckingham $\pi$ theorem is a well established method to reduce the number of experimental variables [5-9]. Numbers of $\pi$ groups (i.e., dimensionless numbers, e.g., Reynolds number $(\mathrm{Re})$, Nusselt number $(\mathrm{Nu})$, Weber number $(\mathrm{We}))$ are based on the degrees of freedom between the problem's quantities and primary units. Huang et al. [5] presented a few examples to show how the Buckingham $\pi$ theorem can be used to match the physical-scale with the lattice-scale. In a capillary rise example, they set a capillary slit's width of $0.002 \mathrm{~m}$. Water with a density $\rho_{\text {water }}=1000 \mathrm{~kg} / \mathrm{m}^{3}$ and a surface tension $\sigma=72.13 \times 10^{-3} \mathrm{~N} / \mathrm{m}$ is overlain by air with a density $\rho_{\text {air }}=1.23 \mathrm{~kg} / \mathrm{m}^{3}$. They estimated the Bond number $(B o)$ to be $1 / 7.36$. The Bo number is

$$
\text { Bo }=\frac{r^{2}\left(\rho_{\text {water }}-\rho_{\text {air }}\right) g}{\sigma},
$$

where $g=9.8 \mathrm{~m} / \mathrm{s}^{2}$.

In their LBM simulation, they assumed that the system is at a temperature of $0.177 \mathrm{tu}$, where tu is the unit of temperature in the lattice-scale. The Redlich-Kwong (R-K) Equation of State (EoS) was used and they found that the corresponding coexisting densities of that temperature are 5.44 and $0.81 \mathrm{mu} / \mathrm{lu}^{3}$ of water and air, respectively. mu and lu are the units of mass and length in the lattice-scale, respectively. Using the Shan-Chen (SC) [6,7] model, the corresponding surface tension is estimated to be $\sigma=0.096$ when $\tau=1$, where $\tau$ is the dimensionless relaxation time. Accordingly, they estimated the gravity to be $g=3.84 \times 10^{-6} \mathrm{lu} / \mathrm{ts}^{2}$, where ts is the unit of time in the lattice-scale. They assumed that the maximum capillary rise is $200 \mathrm{lu}$, while the LBM simulation predicts $218.4 \mathrm{lu}$. They stated that the discrepancy is due to the compressibility in the SC [6,7] model.

It is not easy to find a required number of dimensionless numbers to map the quantities' units from/to the physical-scale to/from the lattice-scale for some problems by the Buckingham $\pi$ theorem. Moreover, as seen in the above example, the Buckingham $\pi$ theorem method depends on assuming some parameters' values, which is increase the numerical effort. This effort appears in increasing the trials that needed to achieve a converge numerical solution, in other words, finding the appropriate values for assumed parameters. Therefore, the second method, i.e., the principle of corresponding states, comes to the picture to deal with those issues. The principle of corresponding states is also called the scaling method. In most numerical simulations the governing equations are known. Therefore, the non-dimensionalization process is to set a list of references for the variables (i.e., length, velocity, temperature, pressure, etc.). Those references must be known and 
constants. For instance in the multiphase problems, a critical state of density, volume, temperature, and pressure is taken as a reference to non-dimensionalize the property and reduce the number of parameters. For instance, the R-K EoS is as follows:

$$
P=\frac{\rho R T}{1-b \rho}-\frac{a \rho^{2}}{\sqrt{T}(1+b \rho)} .
$$

The equation includes six unknown parameters ( $P$ is pressure, $\rho$ is density, $T$ is temperature, $R$ is gas constant, $a$ is a parameter characterizing the attraction of gas particles, and $b$ is effectively a minimum molar volume [8,9]). The variables $a$ and $b$ can be found by taking the first and second derivatives of the R-K EoS with respect to density at the critical state and equating them to zero. Solving the obtained system of equations yields:

$$
a=\frac{0.42748 R^{2} T_{c}^{2.5}}{P_{\mathcal{C}}}=1.28157 \frac{R T_{c} \sqrt{T_{c}}}{\rho_{\mathcal{c}}},
$$

and

$$
b=0.08664 \frac{R T_{\mathcal{c}}}{P_{c}}=\frac{20}{77 \rho_{\mathcal{c}}},
$$

where the subscript $c$ refers to the critical state. Using a critical state of each property yields the following dimensionless variables:

$$
P_{r}=\frac{P}{P_{c}}, T_{r}=\frac{T}{T_{c}}, \text { and } \rho_{r}=\frac{\rho}{\rho_{c}},
$$

where the subscript $r$ refers to the reduced state.

By substituting Equations (3)-(5) into Equation (2), the R-K EoS becomes:

$$
P_{r}=\frac{T_{r} \rho_{r}}{0.33356-0.08664 \rho_{r}}-\frac{\rho_{r}{ }^{2}}{\sqrt{T_{r}}\left(0.26028+0.067604 \rho_{r}\right)} .
$$

The number of unknown parameters is reduced to half. It is worth mentioning that the $\mathrm{R}-\mathrm{K}$ EoS is one of the most accurate EoS, which is adequate for the calculation of gas-phase properties when $\frac{P}{P_{c}}<\frac{T}{2 T_{c}}$ [10].

A flow in a lid-driven cavity is another example, where the lid velocity $\left(u_{l i d}\right)$ is used to scale the velocity field; the height of the cavity $(H)$ is used to scale the length, etc. The non-dimensional governing equation can be written as:

$$
\frac{\partial U}{\partial t^{*}}+U . \nabla U=-\Delta P+\frac{1}{R e} \nabla^{2} U,
$$

where $t^{*}$ is non-dimensional time scaled by $H / u_{l i d}$, and $R e=u_{l i d} H / v$.

However, another example, utilizing natural convection in a differentially heated cavity, the velocity scale can be defined as either the kinematic viscosity $(v)$ divided by the length scale or the thermal diffusion coefficient $(\alpha)$ divided by the length scale. The open literature is full of examples of non-dimensionalized equations using the above-mentioned method. Note, the proper scaling is different than non-dimensionalizing. However, for numerical simulation, it does matter.

However, there are no reference states of some properties (e.g., specific heat capacity, viscosity, thermal diffusivity, coefficient of expansion, enthalpy, etc.). Usually, the researchers $[5,11-16]$ couple the Buckingham $\pi$ theorem with the principle of corresponding states (scaling) method to overcome the mentioned disadvantages. Huang et al. [17] mentioned that not every equation or variable could be easily converted by the Buckingham $\pi$ theorem or scaling method. Consequently, they [17] tried to solve the whole problem by utilizing the Planck unit system. They tested their approach on a $2 \mathrm{D}$ convective heat transfer problem in tube banks. They used three unit systems for mapping properties; physical, Planck, and lattice unit systems. The approach is complicated and has some 
issues. The complication appears on their methodology to represent the variables on the unit systems. Each variable was represented seven times: once in a physical system, thrice in the Planck system, and thrice in a lattice system. For instance, the length was represented as lattice length in the physical, Planck, and lattice unit systems; Planck length in the physical, Planck, and lattice unit systems; and physical length in the physical unit system. In practice, it is preferred to solve some physical problems in the non-dimensional space. However, their approach does not provide this option, as the Planck system was used for scaling.

The literature review shows that the Buckingham $\pi$ theorem and scaling are the common methods used for transferring quantities' units between scales. However, those methods suffer some issues, such as unable to find a required number of dimensionless numbers for the mapping process and there are no reference states of some properties for the scaling process. The current article proposes a dimensional analysis approach to resolve those mentioned issues, where the scaling is performed based on the primary units. Table 1 summarizes the primary dimensions in SI and lattice system units. The proposed approach systematically transforms the quantities' units from/to the physical-scale to/from the lattice-scale. It is known that the LBM is a pseudo-compressible method, which simulates incompressible flows by having a small Mach number to ensure the fluctuations of density in time are not that big. Therefore, the imposed velocity should be selected to ensure a low Mach number. In addition, the stability of the LBM is related to the relaxation time $(\tau)$, where $v=(\tau-0.5) C_{s}^{2} \Delta t\left(C_{s}\right.$ is the pseudo-speed of sound of the lattice). Therefore, $\tau$ is restricted to be higher than 0.5 to ensure the positivity of the viscosity $(v)$. The proposed method has the flexibility to ensure the stability and accuracy of LBM by selecting proper mapping parameters.

Table 1. Primary dimensions in SI and lattice system units.

\begin{tabular}{ccc}
\hline Primary Dimension & SI Unit & Lattice Unit \\
\hline Mass $\{M\}$ & kilogram $(\mathrm{kg})$ & mass unit $(\mathrm{mu})$ \\
Length $\{L\}$ & meter $(\mathrm{m})$ & length unit $(\mathrm{lu})$ \\
Time $\{t\}$ & second $(\mathrm{s})$ & time unit $(\mathrm{ts})$ \\
Temperature $\{\Theta\}$ & Kelvin $(\mathrm{K})$ & temperature unit $(\mathrm{tu})$ \\
\hline
\end{tabular}

In the following section, the proposed method of mapping quantities' units is presented and discussed with supporting example. Section 3 presents and discusses the results of the current work. Six benchmark examples are presented to evaluate the proposed approach. Finally, Section 4 summarizes the conclusions.

\section{Unit Conversion from/to Physical-Scale to/from Lattice-Scale}

In the following, the proposed approach is presented and explained. Firstly, reference parameters that are used to non-dimensionalize the Boltzmann transport equation and any property are inferred. Then, a step-by-step example is performed to illustrate the concept using the inferred reference parameters.

\subsection{Boltzmann Transport Equation and Its Dimensionless Form}

The Bhatnagar-Gross-Krook (BGK) [18] Boltzmann transport equation can be written as $[4,19-22]$ :

$$
\frac{\partial f}{\partial t}+\boldsymbol{e} \cdot \nabla f+\boldsymbol{a} \cdot \nabla_{e} f=\frac{1}{\lambda}\left(f^{M}-f\right),
$$

where $f=f(\boldsymbol{x}, \boldsymbol{e}, t)$ is the particle distribution function, $\boldsymbol{e}$ is the microscopic velocity vector, $a$ is the acceleration, $\lambda$ is the relaxation time, and $f^{M}$ is the Maxwell-Boltzmann distribution function which is given as $[4,19-22]$

$$
f^{M}=\frac{\rho}{(2 \pi R T)^{D / 2}} \exp \left[\frac{-\{(\boldsymbol{e}-\boldsymbol{u}) \cdot(\boldsymbol{e}-\boldsymbol{u})\}}{2 R T}\right],
$$


where $\rho$ is the density, $u$ is the macroscopic velocity, $R$ is the gas constant, $T$ is the temperature, and $D$ is the number of spatial dimensions.

The particle distribution function $(f)$ has the same unit of $f^{M}$. The exponential term in Equation (9) is non-dimensional. Therefore, the unit of the $f$ is the unit of $\frac{\rho}{(R T)^{D / 2}}$. Using dimensional analysis yields:

$$
\{f\}=\left\{M \cdot L^{-3} \cdot t^{D} \cdot L^{-D}\right\} .
$$

It is interesting to notice that the unit of $f$ gives a physical insight about its meaning. The $f$ is density in a hyperspace. The density can be found by using the multiple integrals of $f$ over the velocity

$$
\rho=\int f d e,
$$

where the integration is single, double, or triple for 1D, 2D, or 3D problems, respectively.

Wolf-Gladrow [19] used the characteristic length scale, the reference speed, the reference density, and the time between particle collisions to non-dimensionalize the Boltzmann transport equation, Equation (8). The current work uses unknown reference basic parameters to non-dimensionalize Equation (8) as:

$$
\frac{\partial f^{*}}{\partial t^{*}}+e^{*} \cdot \nabla^{*} f^{*}+a^{*} \cdot \nabla_{e}^{*} f^{*}=\frac{1}{\lambda^{*}}\left(f^{M, *}-f^{*}\right),
$$

where

$$
\begin{gathered}
f^{*}=f \frac{x_{0}^{3}}{m_{o}} \frac{x_{o}^{D}}{t_{o}^{D}}, \\
t^{*}=\frac{t}{t_{o}} \\
\boldsymbol{e}^{*}=\boldsymbol{e} \frac{t_{o}}{x_{o}}, \\
\nabla^{*}=x_{o} \nabla, \\
\boldsymbol{a}^{*}=\boldsymbol{a} \frac{t_{0}^{2}}{x_{o}} \\
\nabla_{e}^{*}=\frac{x_{o}}{t_{o}} \nabla_{e},
\end{gathered}
$$

and

$$
\lambda^{*}=\lambda / t_{0},
$$

where $m_{0}, x_{0}$, and $t_{0}$ are unknown references of mass, length, and time, respectively. Those basic parameters are chosen based on the problem to be solved, as will be presented in the upcoming section. Moreover, those basic parameters including temperature, $\Theta_{0}$, can be used to non-dimensionalize the controlling parameters, such as density, velocity, specific heat capacity, viscosity, etc. The present work's primary key is to take advantage of transferring the units from one-unit scale to another using the basic reference parameters. Hence, we will have better control of the relaxation time and characteristic velocity of the LBM, which improves the accuracy and stability of the simulation of the given problem. In the following section, the methodology of converting the units of quantities from/to the physical-scale to/from the lattice-scale will be explained in detail.

In order to solve Equation (8) numerically, the discretized form is used:

$$
f_{i}\left(\boldsymbol{x}+\boldsymbol{e}_{i} \Delta t, t+\Delta t\right)=f_{i}(\boldsymbol{x}, t)+\frac{1}{\tau}\left(f_{i}^{e q}(\boldsymbol{x}, t)-f_{i}(\boldsymbol{x}, t)\right)+\Delta t S_{i}(\boldsymbol{x}, t),
$$

where $\boldsymbol{e}_{i}$ is discrete lattice velocity, $\Delta t$ is the time step, $\tau=\frac{\lambda}{\Delta t}$ is the dimensionless relaxation time, $f_{i}^{e q}$ is the Maxwell-Boltzmann equilibrium distribution function, and $S_{i}$ is the lattice 
force term. Recently, we [11] reviewed and evaluated twelve force terms that proposed to implement the momentum forces in the LBM.

\subsection{Methodology of Quantities Converting}

Converting the quantities' units of a certain problem from/to physical-scale to/from lattice-scale is not difficult. However, it needs careful treatment that ensures a correct and consistent scaling of all quantities. Thus, the current approach suggests using basic reference parameters of the physical-scale and lattice-scale in the converting process. Figure 2 shows a flowchart of the unit conversion methodology of the present approach. The basic reference parameters of the physical-scale $\left(x_{o(P S)}, t_{o(P S)}, m_{o(P S)}\right.$, and $\left.\Theta_{o(P S)}\right)$ are applied to convert properties units to the dimensionless-scale. Those properties at the dimensionless-scale are converted to the lattice-scale using the basic reference parameters of the lattice-scale $\left(x_{o(L S)}, t_{o(L S)}, m_{o(L S)}\right.$, and $\left.\Theta_{o(L S)}\right)$ and vice versa.

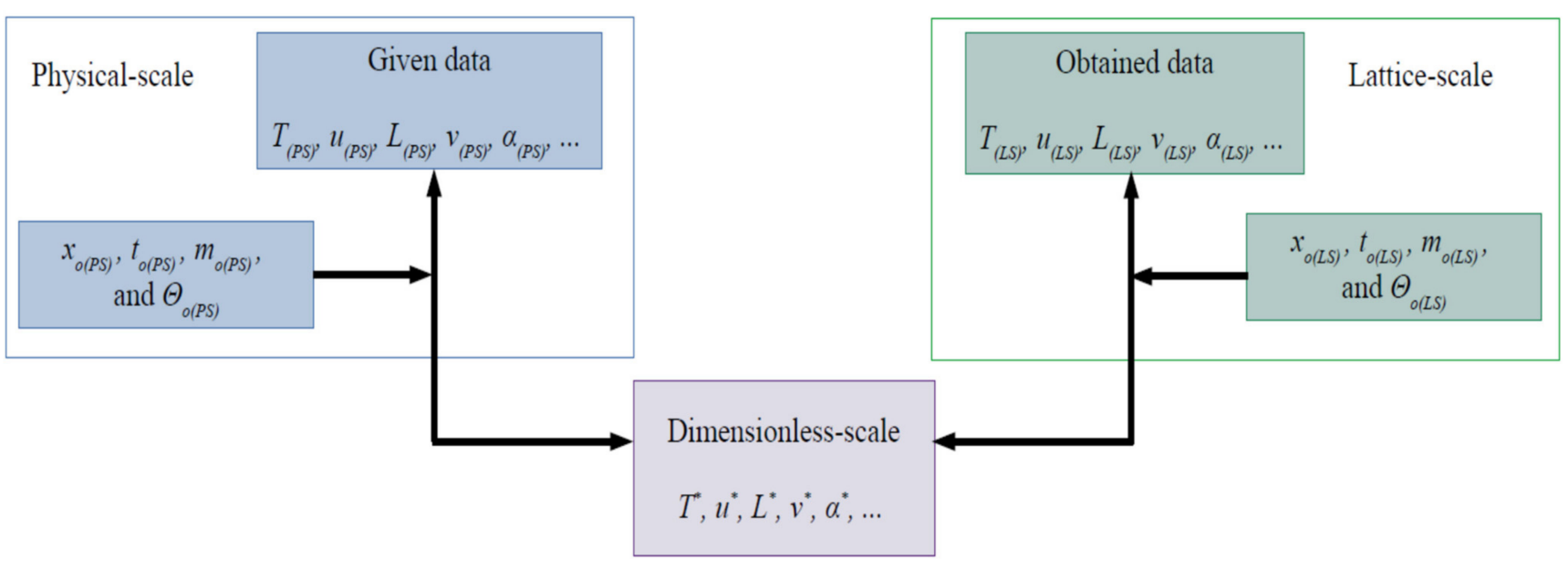

Figure 2. Flowchart of unit conversion approach.

It is better to illustrate the methodology with an example. Assume natural convection in a cavity filled with air at room temperature $(298 \mathrm{~K})$ has $0.02 \mathrm{~m}$ and $0.04 \mathrm{~m}$ in high $(\mathrm{H})$ and in length (L), respectively. The air properties at room temperature are $1.2 \mathrm{~kg} / \mathrm{m}^{3}$ of density $(\rho), 1.56 \times 10^{-5} \mathrm{~m}^{2} / \mathrm{s}$ of viscosity $(v), 2.14 \times 10^{-5} \mathrm{~m}^{2} / \mathrm{s}$ of thermal diffusivity $(\alpha)$, and $3.4 \times 10^{-3} \mathrm{~K}^{-1}$ of the coefficient of expansion $(\beta)$. The west wall is heated to a temperature of $500 \mathrm{~K}$, while the east wall is kept at room temperature. The bottom and upper boundaries are subjected to adiabatic conditions. Figure 3 displays the schematic diagram of the problem.

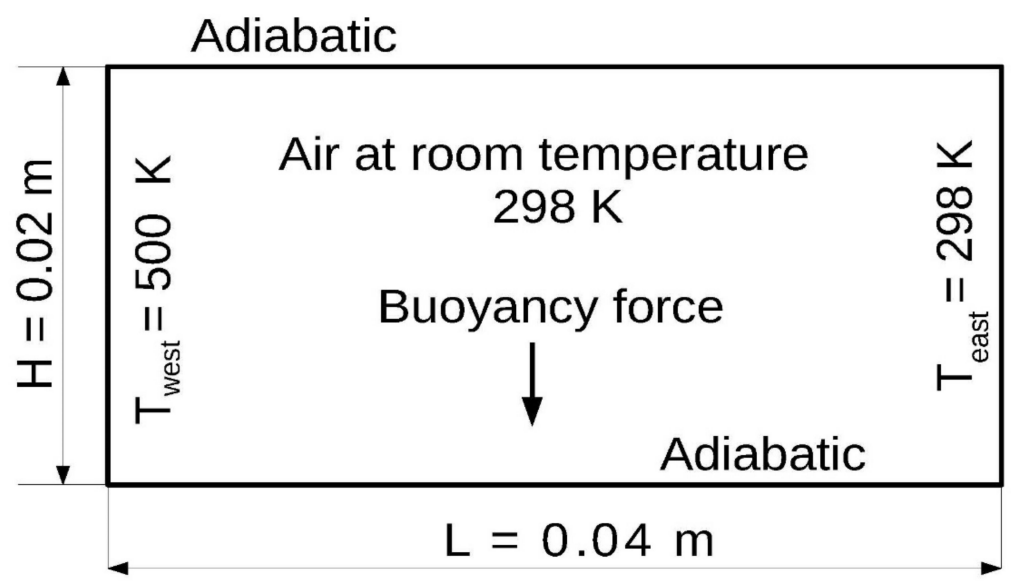

Figure 3. Schematic diagram of the differentially heated cavity. 
The dimensionless governing equations (Navier-Stokes equations) for natural convection can be written as:

Continuity equation:

$$
\frac{\partial U}{\partial X}+\frac{\partial V}{\partial Y}=0
$$

$\mathrm{x}$-momentum equation:

$$
U \frac{\partial U}{\partial X}+V \frac{\partial U}{\partial Y}=-\frac{\partial P}{\partial X}+\frac{1}{R e}\left(\frac{\partial^{2} U}{\partial X^{2}}+\frac{\partial^{2} U}{\partial Y^{2}}\right)
$$

y-momentum equation:

$$
U \frac{\partial V}{\partial X}+V \frac{\partial V}{\partial Y}=-\frac{\partial P}{\partial Y}+\frac{1}{R e}\left(\frac{\partial^{2} V}{\partial X^{2}}+\frac{\partial^{2} V}{\partial Y^{2}}\right)+\frac{G r}{R e^{2}} \theta, \text { and }
$$

Energy equation:

$$
U \frac{\partial \theta}{\partial X}+V \frac{\partial \theta}{\partial Y}=\frac{1}{\operatorname{Re} P r}\left(\frac{\partial^{2} \theta}{\partial X^{2}}+\frac{\partial^{2} \theta}{\partial Y^{2}}\right)
$$

where

$$
\begin{gathered}
X=\frac{x}{H}, Y=\frac{y}{H^{\prime}} \\
U=\frac{u}{u_{0}}, V=\frac{v}{u_{o}}, \\
P=\frac{p}{\rho u_{o}^{2}} \text {, and } \theta=\frac{T-T_{\text {west }}}{T_{\text {east }}-T_{\text {west }}} .
\end{gathered}
$$

The controlling parameters for the example are summarized in Table 2. As the LBM is a pseudo-compressible method, the magnitude of $u$ should be ensured that the Mach number is within the limit of incompressible flow $(M a<0.3)$. The formulas and values of $u$ and $M a$ are listed in Table 2.

Table 2. Parameters and dimensionless numbers of the example. $g=9.81 \mathrm{~m} / \mathrm{s}^{2}$ is the gravity and $\mathrm{C}=343 \mathrm{~m} / \mathrm{s}$ is the speed of sound.

\begin{tabular}{ccc}
\hline Parameter & Formula & Value \\
\hline Prandtl number & $\operatorname{Pr}=\frac{v}{\alpha}$ & 0.73 \\
\hline Grashof number & $G r=\frac{g \beta \Delta T H^{3}}{v^{2}}$ & $2.2145 \times 10^{5}$ \\
\hline Reynold number & $R e=\sqrt{G r}$ & 470.6196 \\
\hline Velocity & $u=\sqrt{g \beta \Delta T H}$ & $0.3671 \mathrm{~m} / \mathrm{s}$ \\
\hline Mach number & $M a=\frac{u}{C}$ & $1.07 \times 10^{-3}$ \\
\hline
\end{tabular}

To convert the problem to the lattice-scale, the next steps should be followed:

Physical-scale: In the data reduction process, there is a need to select the basic reference variables of the physical-scale of the problem as the characteristic scales. Therefore, $x_{o(P S)}=0.02 \mathrm{~m}$ and $\Theta_{o(P S)}=500 \mathrm{~K}$ are chosen as the reference variables for length and temperature, respectively. Furthermore, the reference of time $\left(t_{o(P S)}\right)$ and mass $\left(m_{o(P S)}\right)$ scales can be estimated by selecting the dimensionless parameters, $\rho^{*}$ and $u^{*}$, which are the dimensionless density and velocity, respectively. As free parameters, any values for $\rho^{*}$ and $u^{*}$ can be selected. Let us set $\rho^{*}=u^{*}=1$ for simplicity. The subscript PS refers to the physical-scale and the superscript $*$ refers to the dimensionless-scale. Using the dimensionless density and velocity, the other reference parameters $\left(m_{o(P S)}\right.$ and $\left.t_{o(P S)}\right)$ can be calculated as:

$$
m_{o(P S)}=\frac{\rho}{\rho^{*}} x_{o(P S)}^{3}=9.6 \times 10^{-6} \mathrm{~kg},
$$


and

$$
t_{o(P S)}=\frac{u^{*}}{u} x_{o(P S)}=0.0545 \mathrm{~s} .
$$

Dimensionless-scale: Using the mentioned reference variables of the physical-scale, the non-dimensionalized parameters are as follows:

$$
\begin{gathered}
H^{*}=\frac{H_{(P S)}}{x_{o(P S)}}=1, \\
L^{*}=\frac{L_{(P S)}}{x_{o(P S)}}=2, \\
C^{*}=C \frac{t_{o(P S)}}{x_{o(P S)}}=934.3928, \\
v^{*}=v_{(P S)} \frac{t_{o(P S)}}{x_{o(P S)}^{2}}=2.12 \times 10^{-3}, \\
\alpha^{*}=\alpha_{(P S)} \frac{t_{o(P S)}}{x_{o(P S)}^{2}}=2.91 \times 10^{-3}, \\
g^{*}=g_{(P S)} \frac{t_{o(P S)}^{2}}{x_{o(P S)}}=1.456, \\
\beta^{*}=\beta_{(P S)} \Theta_{o(P S)}=1.7, \\
\Theta_{w e s t}^{*}=\frac{\Theta_{w e s t(P S)}}{\Theta_{o(P S)}}=1,
\end{gathered}
$$

and

$$
\Theta_{\text {east }}^{*}=\Theta_{\text {south }}^{*}=\frac{\Theta_{\text {east }(P S)}}{\Theta_{o(P S)}}=0.596 .
$$

The dimensionless numbers will be

$$
\begin{gathered}
\operatorname{Pr}=\frac{v^{*}}{\alpha^{*}}=0.73, \\
G r=\frac{g^{*} \beta^{*} \Delta \Theta^{*} H^{*^{3}}}{v^{*^{2}}}=2.2145 \times 10^{5}, \\
\operatorname{Re}=\sqrt{G r}=470.6196,
\end{gathered}
$$

and

$$
M a=\frac{u^{*}}{C^{*}}=1.07 \times 10^{-3} .
$$

Lattice-scale: The reference variables of the lattice-scale $\left(\Theta_{o(L S)}, m_{o(L S)}, x_{o(L S)}\right.$, and $\left.t_{o(L S)}\right)$ can be calculated. Notice that the formula of each non-dimensional parameter expressed on the physical-scale, i.e., Equations (28)-(38), should be similar to that expressed on the lattice-scale. Hence,

$$
\begin{gathered}
\rho^{*}=\rho_{(L S)} \frac{x_{o(L S)}^{3}}{m_{o(L S)}}=1, \\
u^{*}=u_{(L S)} \frac{t_{o(L S)}}{x_{o(L S)}}=1, \\
v^{*}=v_{(L S)} \frac{t_{o(L S)}}{x_{o(L S)}^{2}}=2.12 \times 10^{-3},
\end{gathered}
$$




$$
\alpha^{*}=\alpha_{(L S)} \frac{t_{o(L S)}}{x_{o(L S)}^{2}}=2.91 \times 10^{-3},
$$

and

$$
\Theta_{\text {west }(L S)}^{*}=\frac{\Theta_{\text {west }(L S)}}{\Theta_{o(L S)}}=1 .
$$

where the subscript $L S$ refers to the lattice-scale. Again, we have two free parameters, $\rho_{(L S)}$ and $\Theta_{o(L S)}$. Any reasonable values can be selected, usually set to be one. Other scales, $m_{o(L S)}, x_{o(L S)}$, and $t_{o(L S)}$ can be found by manipulating Equations (43)-(47):

$$
\begin{gathered}
m_{o(L S)}=\frac{x_{o(L S)}^{3}}{\rho^{*}} \rho_{(L S)} \\
t_{o(L S)}=\frac{v_{(L S)}}{v^{*}}\left(\frac{u^{*}}{u_{(L S)}}\right)^{2}=\frac{\alpha_{(L S)}}{\alpha^{*}}\left(\frac{u^{*}}{u_{(L S)}}\right)^{2}=\frac{u^{*}}{u_{(L S)}} x_{o(L S)},
\end{gathered}
$$

and

$$
x_{o(L S)}=\frac{v_{(L S)}}{v^{*}} \frac{u^{*}}{u_{(L S)}}=\frac{\alpha_{(L S)}}{\alpha^{*}} \frac{u^{*}}{u_{(L S)}}=\frac{u_{(L S)}}{u^{*}} t_{o(L S)} .
$$

The reference lattice velocity $\left(u_{(L S)}\right)$, lattice viscosity $\left(v_{(L S)}\right)$, and lattice thermal diffusivity $\left(\alpha_{(L S)}\right)$ are free parameters. They should be selected to satisfy stability and accuracy. It is well known that the stability of the LBM is related to the relaxations time $\left(\tau_{f}\right.$ and $\left.\tau_{e}\right)$, where $v_{(L S)}=\left(\tau_{f}-0.5\right) C_{S}^{2} \Delta t$ and $\alpha_{(L S)}=\left(\tau_{e}-0.5\right) C_{S}^{2} \Delta t$. The subscripts $f$ and $e$ refer to the momentum and energy equations, respectively. $C_{s}$ is the pseudo-speed of sound of the lattice. The pseudo-speed of sound is the maximum speed needed to transfer data in the lattice, which is a function of the lattice model, for D2Q9, is equal to $\frac{1}{\sqrt{3}}$. Hence, the values of $\tau_{f}$ and $\tau_{e}$ should not be close to 0.5 to satisfy stability. The restriction imposed on the lattice velocity $\left(u_{(L S)}\right)$ is that $M a_{(L S)}=u_{(L S)} / C_{S}<<1 . M a_{(L S)}$ is the lattice Mach number in the lattice-scale, which is different from the physical Mach number.

As mentioned before, the stability condition of LBM is that $\tau_{f}$ and $\tau_{e}$ should be greater than 0.5 . Let $\tau_{f}=1$. For incompressibility condition the Ma number should be small. Let $u_{(L S)}=0.1$, which yields $M a_{(L S)}$ of 0.1732 , which is $<<1$. Hence, Equations (48)-(50) yield:

$$
\begin{gathered}
v_{(L S)}=\frac{1-0.5}{3}=0.1667 \mathrm{lu}^{2} / \mathrm{ts}, \\
t_{o(L S)}=\frac{0.1667}{2.12 \times 10^{-3}} \times\left(\frac{1}{0.1}\right)^{2}=7.84 \times 10^{3} \mathrm{ts},
\end{gathered}
$$

and

$$
x_{o(L S)}=\frac{0.1667}{2.12 \times 10^{-3}} \times \frac{1}{0.1}=784.3661 \mathrm{lu} .
$$

The relaxation time related to the energy equation can be calculated as:

$$
\alpha_{(L S)}=784.3661 \times 2.91 \times 10^{-3} \times \frac{0.1}{1}=0.2286 \mathrm{lu}^{2} / \mathrm{ts},
$$

then

$$
\tau_{e}=\frac{\alpha_{(L S)}}{C_{S}^{2} \Delta t}+0.5=1.1859 .
$$

Finally, the parameters of the problem in the lattice-scale will be:

$$
\begin{aligned}
& H_{(L S)}=x_{o(L S)} H^{*}=784.3661 \mathrm{lu}, \\
& L_{(L S)}=x_{o(L S)} L^{*}=1568.7321 \mathrm{lu},
\end{aligned}
$$




$$
\begin{gathered}
C_{(L S)}=C \frac{x_{o(L S)}}{t_{o(L S)}}=93.4393 \mathrm{lu} / \mathrm{ts}, \\
g_{(L S)}=g^{*} \frac{x_{o(L S)}}{t_{o(L S)}^{2}}=1.86 \times 10^{-5} \mathrm{lu} / \mathrm{ts}^{2}, \\
\beta_{(L S)}=\frac{\beta^{*}}{\Theta_{o(L S)}}=1.7 \mathrm{tu}^{-1}, \\
\Theta_{\text {west }(L S)}=\Theta_{\text {west }}^{*} \Theta_{o(L S)}=1 \mathrm{tu},
\end{gathered}
$$

and

$$
\Theta_{\text {east }(L S)}=\Theta_{\text {south }(L S)}=\Theta_{\text {south }}^{*} \Theta_{o(L S)}=0.596 \mathrm{tu} .
$$

To check the similarity, the dimensionless numbers can be calculated as:

$$
\begin{gathered}
\operatorname{Pr}=\frac{\nu_{(L S)}}{\alpha_{(L S)}}=0.73, \\
G r=\frac{g_{(L S)} \beta_{(L S)} H_{(L S)}^{3} \Delta \Theta_{(L S)}}{v_{(L S)}^{2}}=2.2145 \times 10^{5}, \\
\operatorname{Re}=\sqrt{G r}=470.6196,
\end{gathered}
$$

and

$$
\begin{gathered}
M a=\frac{u_{(L S)}}{C_{(L S)}}=1.07 \times 10^{-3}, \\
M a_{(L S)}=\frac{u_{(L S)}}{C_{S}}=0.1732,
\end{gathered}
$$

One advantage of the method is freedom of selection $\tau_{f}$ or $\tau_{e}$ to ensure the stability of the solution and in selecting $u_{(L S)}$ to ensure the incompressibility conditions.

The scaling units between SI and lattice unit systems can be summarized in Table 3. The values listed in Table 3 are calculated by dividing each reference variable by its corresponding value in physical-scale (SI unit). The Appendix A gives the factors to convert quantities from/to physical-scale to/from dimensionless-scale from/to lattice-scale.

Table 3. Scaling units between SI and lattice systems.

\begin{tabular}{ccc}
\hline Primary Dimension & SI Unit & Lattice Unit \\
\hline Mass & $1 \mathrm{~kg} / \mathrm{kg}$ & $5.027 \times 10^{13} \mathrm{mu} / \mathrm{kg}$ \\
Length & $1 \mathrm{~m} / \mathrm{m}$ & $3.9218 \times 10^{4} \mathrm{lu} / \mathrm{m}$ \\
Time & $1 \mathrm{~s} / \mathrm{s}$ & $1.4396 \times 10^{5} \mathrm{ts} / \mathrm{s}$ \\
Temperature & $1 \mathrm{~K} / \mathrm{K}$ & $2.0 \times 10^{-3} \mathrm{tu} / \mathrm{K}$ \\
\hline
\end{tabular}

\section{Results and Discussions}

To evaluate the feasibility and accuracy of the proposed approach, one- and twodimensional benchmark examples whose analytical or numerical solutions available are performed. The D1Q3 lattice model is used to solve one-dimensional problems, while the D2Q5 and D2Q9 for two-dimensional. Figure 4 shows the index and direction of each microscopic velocity vector in the used lattice models.

\subsection{One-Dimensional Examples}

The solution of 1D diffusion, 1D advection-diffusion, and 1D phase change problems are provided to exemplify the capacity of the proposed approach. The D1Q3 lattice model is 
used to solve those examples, where it has the following equilibrium distribution function $\left(f_{i}^{e q}\right)$, lattice weights $\left(w_{i}\right)$, microscopic speeds $\left(\boldsymbol{e}_{i}\right)$, and speed of sound $\left(C_{S}\right)$ :

$$
\begin{gathered}
f_{i}^{e q}=w_{i} \phi\left[1+\frac{\boldsymbol{e}_{i} \cdot \boldsymbol{u}^{e q}}{C_{s}^{2}}\right], \\
w_{i}=\left[\begin{array}{lll}
4 / 6 & 1 / 6 & 1 / 6
\end{array}\right], \\
\boldsymbol{e}_{i}=\left[\begin{array}{lll}
0 & 1 & -1
\end{array}\right],
\end{gathered}
$$

and

$$
C_{s}^{2}=\frac{1}{3}
$$
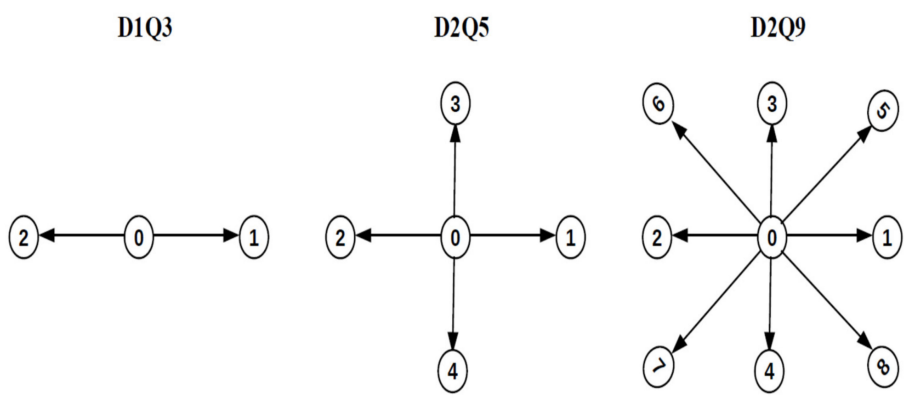

Figure 4. Schematic diagram of D1Q3, D2Q5, and D2Q9 lattice models.

\subsubsection{D Diffusion}

In this example, an insulated rod of copper whose left and right ends are maintained at constant temperatures of $298 \mathrm{~K}$ and $373 \mathrm{~K}$, respectively. The rod has length $L=2 \mathrm{~m}$ and uniform heat generation $q=100 \mathrm{~kW} / \mathrm{m}^{3}$. The properties of the copper in the SI unit system are listed in Table 4 . The problem is governed by the following equation

$$
\rho C_{p} \frac{\partial T}{\partial t}=k \frac{\partial^{2} T}{\partial x^{2}}+q .
$$

where $C_{p}$ is the specific heat at constant pressure and $k$ is the thermal conductivity.

The analytical solution of the steady-state temperature distribution is given as Ref. [23]:

$$
T=\left[\frac{T_{c}-T_{h}}{L}+\frac{q}{2 k}(L-x)\right] x+T_{h} .
$$

where $T_{c}$ and $T_{h}$ are the cold and hot temperatures, respectively.

In LBM simulation, the following data are used:

$$
\tau_{e}=1,
$$

and

$$
S_{i}=w_{i} \frac{q}{\rho C_{p}} .
$$

Using the previously mentioned steps in Section 2.2, each quantity can be mapped from the physical-scale to the dimensionless-scale after the reference variables of the physicalscale are defined. The current example is characterized by stability only. This gives more flexibility in choosing the length reference variable for the lattice-scale. Based on that $\tau_{e}=1$ and $x_{o(L S)}=10 \mathrm{lu}$ are selected. By setting $\rho_{(L S)}=\Theta_{o(L S)}=1$ and manipulating Equations (48)-(50), all of the reference variables of the lattice-scale are defined. Follow Equations (56)-(62), each quantity is mapped from a dimensionless-scale to the lattice-scale. Table 4 shows that all quantities of the current example are mapped from the physical-scale to the lattice-scale using the present approach. Table 4 lists the reference variables of the physical- 
scale and lattice-scale, the scaling units between SI and lattice systems, and the transforming properties between scales. The values with * represent those values are assumed.

Figure 5 shows the temperature distribution along the rod. The steady-state condition is achieved when the difference between two successive iterations of temperature is less than $10^{-9}$. Figure 5 a compares the LBM predictions with analytical solution (i.e., Equation (73)) at the lattice-scale. Figure 5b compares the LBM predictions with the analytical solution at the physical-scale. It can be concluded that excellent agreements between the numerical and analytical solutions at both scales can be achieved.

Table 4. Converting data for diffusion problem. The values with * represent assumed values.

\begin{tabular}{|c|c|c|c|}
\hline \multicolumn{4}{|c|}{ Reference Variables in Physical-Scale and Lattice-Scale } \\
\hline Reference Variables & \multicolumn{2}{|c|}{ Physical-Scale } & Lattice-Scale \\
\hline$m_{0}$ & \multicolumn{2}{|c|}{$71,520 \mathrm{~kg}$} & $1000 \mathrm{mu}$ \\
\hline$x_{0}$ & \multicolumn{2}{|c|}{$2 \mathrm{~m}$} & $10 \mathrm{lu}$ \\
\hline$t_{o}$ & \multicolumn{2}{|c|}{$3.4635 \times 10^{4} \mathrm{~s}$} & 600 ts \\
\hline$\Theta_{0}$ & \multicolumn{2}{|c|}{$373 \mathrm{~K}$} & $1 * \mathrm{tu}$ \\
\hline \multicolumn{4}{|c|}{ Scaling units between SI and lattice systems } \\
\hline Primary dimension & \multicolumn{2}{|c|}{ Physical-scale } & Lattice-scale \\
\hline Mass & \multicolumn{2}{|c|}{$1 \mathrm{~kg} / \mathrm{kg}$} & $0.014 \mathrm{mu} / \mathrm{kg}$ \\
\hline Length & \multicolumn{2}{|c|}{$1 \mathrm{~m} / \mathrm{m}$} & $5 \mathrm{lu} / \mathrm{m}$ \\
\hline Time & \multicolumn{2}{|c|}{$1 \mathrm{~s} / \mathrm{s}$} & $0.017 \mathrm{ts} / \mathrm{s}$ \\
\hline Temperature & \multicolumn{2}{|c|}{$1 \mathrm{~K} / \mathrm{K}$} & $2.68 \times 10^{-3} \mathrm{tu} / \mathrm{K}$ \\
\hline \multicolumn{4}{|c|}{ Converting properties between scales } \\
\hline Property & Physical-scale & Dimensionless-scale & Lattice-scale \\
\hline Length $(L)$ & $2 \mathrm{~m}$ & 1 & $10 * 1 \mathrm{u}$ \\
\hline Cold temperature $\left(T_{\mathcal{C}}\right)$ & $298 \mathrm{~K}$ & 0.799 & $0.799 \mathrm{tu}$ \\
\hline Hot temperature $\left(T_{h}\right)$ & $373 \mathrm{~K}$ & 1 & $1 \mathrm{tu}$ \\
\hline Density $(\rho)$ & $8940 \mathrm{~kg} / \mathrm{m}^{3}$ & $1 *$ & $1 * \mathrm{mu}^{\prime l u} \mathrm{u}^{-3}$ \\
\hline Specific heat $\left(C_{p}\right)$ & $385 \mathrm{~J} / \mathrm{kg} . \mathrm{K}$ & $4.3068 \times 10^{13}$ & $1.19 \times 10^{10} \mathrm{lu}^{2} \cdot \mathrm{ts}^{-2} \cdot \mathrm{tu}^{-1}$ \\
\hline Thermal conductivity $(k)$ & $397.5 \mathrm{~W} / \mathrm{m} . \mathrm{K}$ & $4.3068 \times 10^{13}$ & $1.99 \times 10^{9}$ mu.lu.ts ${ }^{-3} \cdot$ tu $^{-1}$ \\
\hline Thermal diffusivity $(\alpha)$ & $1.1549 \times 10^{-4} \mathrm{~m}^{2} / \mathrm{s}$ & $1 *$ & $0.1667 \mathrm{lu}^{2} . \mathrm{ts}^{-1}$ \\
\hline Uniform heat generation $(q)$ & $1 \times 10^{5} \mathrm{~W} / \mathrm{m}^{3}$ & $1.1619 \times 10^{14}$ & $5.3791 \times 10^{7} \mathrm{mu} \cdot \mathrm{lu}^{-1} \cdot \mathrm{ts}^{-3}$ \\
\hline
\end{tabular}
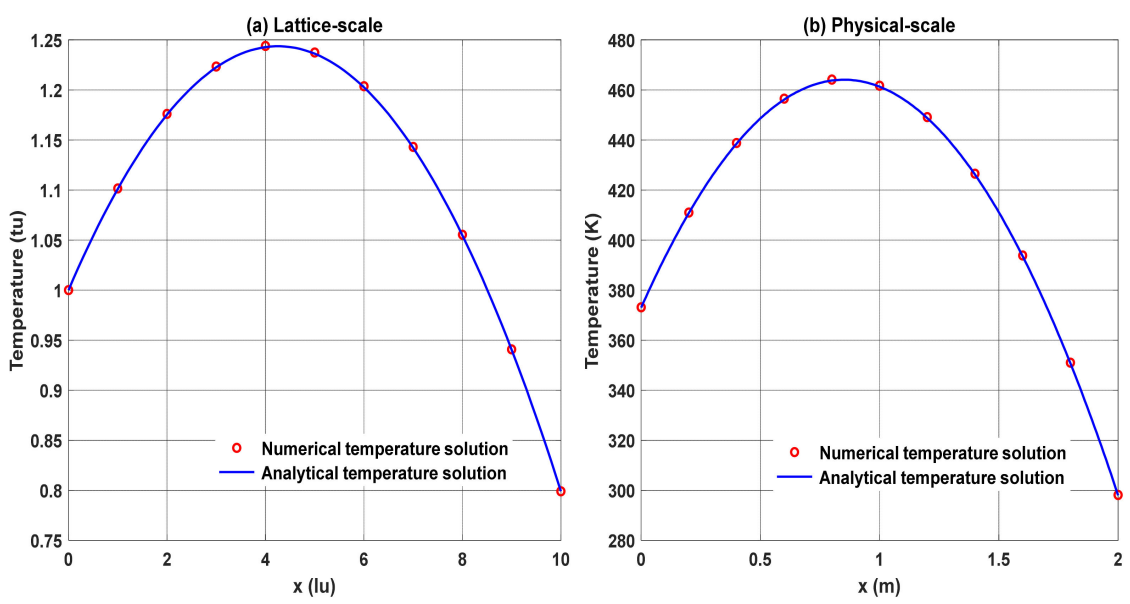

Figure 5. Numerical and analytical solutions of the steady-state temperature distribution along the rod; (a) solutions at the lattice-scale (the analytical solution is achieved using Equation (73) at the lattice-scale), and (b) solutions at the physical-scale (the numerical solution is achieved by the LBM then transformed to the physical-scale using the scaling units from Table 4). 


\subsubsection{D Advection-Diffusion}

In this example, a property $\phi$ is transported by a constant velocity of $0.001 \mathrm{~m} / \mathrm{s}$ by advection-diffusion through the 1D domain. The boundary conditions are $\phi x=0=373 \mathrm{~K}$ and $\phi x=\mathrm{L}=298 \mathrm{~K}$, where $\mathrm{L}=1 \mathrm{~m}$. The property has $1.2 \mathrm{~kg} / \mathrm{m}^{3}$ of density, $1.005 \mathrm{~kJ} / \mathrm{kg} . \mathrm{K}$ of specific heat, and $0.026 \mathrm{~W} / \mathrm{m} . \mathrm{K}$ of thermal conductivity. Due to the advection velocity of the current example is constant, the accuracy criterion does not come to the picture. The $1 \mathrm{D}$ advection-diffusion equation can be expressed as follows:

$$
\rho C_{p} \frac{\partial \phi}{\partial t}+\rho C_{p} u \frac{\partial \phi}{\partial x}=k \frac{\partial^{2} \phi}{\partial x^{2}} .
$$

The analytical solution of the steady-state condition is given by [23]:

$$
\phi=\phi_{x=0}+\left(\phi_{x=L}-\phi_{x=0}\right)\left[\frac{\exp \left(\rho C_{p} u x / k\right)-1}{\exp \left(\rho C_{p} u L / k\right)-1}\right] .
$$

The following data are used in the LBM modeling:

$$
\begin{gathered}
\tau_{e}=1, \\
\boldsymbol{u}^{e q}=\boldsymbol{u}_{(L S)},
\end{gathered}
$$

and

$$
S_{i}=0,
$$

where $\boldsymbol{u}_{(L S)}$ is the advection velocity at the lattice-scale, which is constant.

Figure 6 shows the simulation results for the given example using the listed data in Table 5. Excellent agreements with the analytical solutions at the lattice-scale (Figure 6a) and the physical-scale (Figure $6 \mathrm{~b}$ ) are shown. As mentioned, in such problems, the LBM results are not affected by the advection velocity. Therefore, the lattice Mach number $\left(M a_{(L S)}\right)$ does not affect the results. However, the results of the conventional computational fluid dynamic (CFD) methods are significantly affected by the value of the advection velocity, which causes dispersion error [23]. Figure 7 shows a comparison between the LBM and finite difference method (FDM) at the physical-scale for different mesh sizes. The LBM solution is achieved at the lattice-scale then converted to the physical-scale. As shown, the error between the analytical and FDM solutions is significantly affected by mesh size, unlike the LBM.
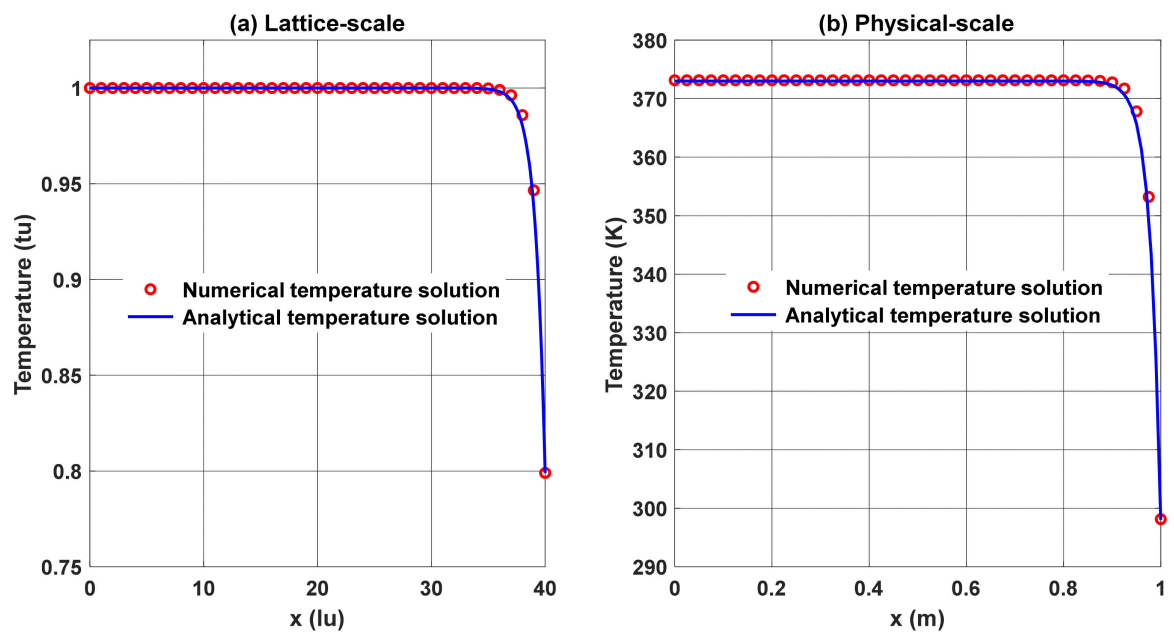

Figure 6. Numerical and analytical solutions of the steady-state temperature distribution along the domain; (a) solutions at the lattice-scale (the analytical solution is achieved using Equation (77) at the lattice-scale), and (b) solutions at the physical-scale (the numerical solution is achieved by the LBM then converted to the physical-scale). 

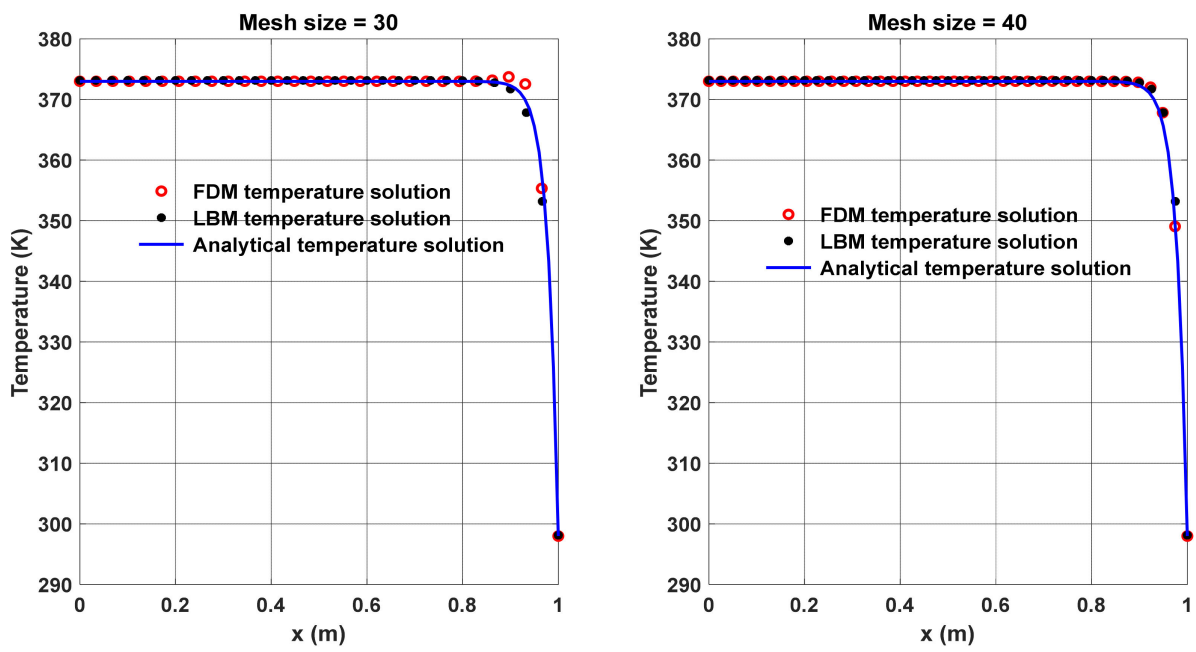

Figure 7. Comparison of the LBM and finite difference method (FDM) for different mesh sizes. The LBM solution is achieved at the lattice-scale then converted to the physical-scale.

Table 5. Converting data for the advection-diffusion problem. The values with * represent assumed values.

\begin{tabular}{|c|c|c|c|}
\hline \multicolumn{4}{|c|}{ Reference Variables in Physical-Scale and Lattice-Scale } \\
\hline Reference Variables & \multicolumn{2}{|c|}{ Physical-Scale } & Lattice-Scale \\
\hline$m_{0}$ & \multicolumn{2}{|c|}{$1.2 \mathrm{~kg}$} & $6.4 \times 10^{4} \mathrm{mu}$ \\
\hline$x_{0}$ & \multicolumn{2}{|c|}{$1 \mathrm{~m}$} & $40 \mathrm{lu}$ \\
\hline$t_{o}$ & \multicolumn{2}{|c|}{$1.0 \times 10^{3} \mathrm{~s}$} & 206.97 ts \\
\hline$\Theta_{0}$ & \multicolumn{2}{|c|}{$373 \mathrm{~K}$} & $1 *$ tu \\
\hline \multicolumn{4}{|c|}{ Converting properties between scales } \\
\hline Property & Physical-scale & Dimensionless-scale & Lattice-scale \\
\hline Length $(L)$ & $1 \mathrm{~m}$ & 1 & $40 * 1 \mathrm{u}$ \\
\hline Advection velocity $(u)$ & $0.001 \mathrm{~m} / \mathrm{s}$ & $1 *$ & 0.1933 lu.ts $^{-1}$ \\
\hline$\phi_{x=L}$ & $298 \mathrm{~K}$ & 0.799 & $0.799 \mathrm{tu}$ \\
\hline$\phi_{x}=0$ & $373 \mathrm{~K}$ & 1 & 1 tu \\
\hline Density $(\rho)$ & $1.2 \mathrm{~kg} / \mathrm{m}^{3}$ & $1 *$ & $1 * \mathrm{mu}^{*} \mathrm{lu}^{-3}$ \\
\hline Specific heat $\left(C_{p}\right)$ & $1005 \mathrm{~J} / \mathrm{kg} . \mathrm{K}$ & $3.75 \times 10^{11}$ & $1.4 \times 10^{10} \mathrm{lu}^{2} \cdot \mathrm{ts}^{-2} \cdot \mathrm{tu}^{-1}$ \\
\hline Thermal conductivity $(k)$ & $0.026 \mathrm{~W} / \mathrm{m} . \mathrm{K}$ & $8.1 \times 10^{9}$ & $2.33 \times 10^{9}$ mu.lu.ts ${ }^{-3} \cdot \mathrm{tu}^{-1}$ \\
\hline Thermal diffusivity $(\alpha)$ & $2.156 \times 10^{-5} \mathrm{~m}^{2} / \mathrm{s}$ & 0.0216 & $0.1667 \mathrm{lu}^{2} \cdot \mathrm{ts}^{-1}$ \\
\hline
\end{tabular}

\subsubsection{D Melting in a Half-Scale (Phase Change)}

A semi-infinite slab of pure aluminum initially is at the uniform melting temperature $\left(660^{\circ} \mathrm{C}\right)$. Suddenly, the left side of the slab $($ at $x=0)$ is subjected to a constant source of a temperature of $750{ }^{\circ} \mathrm{C}$ at times $t \geq 0$. As a result, a melting begins from the left side, where the liquid/solid interface moves in the positive $x$-direction as time progresses. Since the solid phase is at a constant melting temperature, the problem is considered a one-region problem. The temperature distribution in the liquid phase for a given time can be written as Refs. [24,25]:

$$
T=T_{x=0}+\left(T_{x=0}-T_{x=L}\right) \frac{\operatorname{erf}\left(\frac{x}{2 \sqrt{\alpha t}}\right)}{\operatorname{erf}(\lambda)}
$$

where $\lambda$ are solutions to the transcendental equation $\lambda e^{\lambda^{2}} \operatorname{erf}(\lambda)=\frac{S t}{\sqrt{\pi}}$. St $=\frac{C_{p}\left(T_{x=0}-T_{x=L}\right)}{h_{f g}}$ is the Stefan number, where $h_{f g}$ is the latent heat of aluminum. Table 6 summarizes the thermo-physical properties of pure aluminum in the SI unit system [15]. 
For the LBM simulation, Jiaung et al.'s [26] phase change model is used where the following data are used:

$$
\begin{gathered}
\tau_{e}=1, \text { and } \\
S_{i}=-w_{i} \frac{h_{f g}}{C_{p}} \frac{\partial f_{l}}{\partial t},
\end{gathered}
$$

where $f_{l}$ is the volume-phase fraction of the liquid phase and is zero for the solid region and unity for the liquid region.

In the present example, the non-dimensional LBM form is used. Therefore, the dimensionless-scale data listed in Table 6 are applied. Figure 8 displays the temperature distribution along the slab for dimensionless time $t^{*}=1$ (i.e., for $4.6267 \times 10^{2} \mathrm{~s}$ ). The good agreements between the results can be shown in Figure 8 .

\begin{tabular}{|c|c|c|c|}
\hline \multicolumn{4}{|c|}{ Reference Variables in Physical-Scale and Lattice-Scale } \\
\hline Reference Variables & \multicolumn{2}{|c|}{ Physical-Scale } & Lattice-Scale \\
\hline$m_{0}$ & \multicolumn{2}{|c|}{$21.59 \mathrm{~kg}$} & $1 \times 10^{6} \mathrm{mu}$ \\
\hline$x_{0}$ & \multicolumn{2}{|c|}{$0.2 \mathrm{~m}$} & $100 \mathrm{lu}$ \\
\hline$t_{0}$ & \multicolumn{2}{|c|}{$4.6267 \times 10^{2} \mathrm{~s}$} & $6 \times 10^{4}$ ts \\
\hline$\Theta_{0}$ & \multicolumn{2}{|c|}{$1023 \mathrm{~K}$} & $1 *$ tu \\
\hline \multicolumn{4}{|c|}{ Converting properties between scales } \\
\hline Property & Physical-scale & Dimensionless-scale & Lattice-scale \\
\hline Length $(L)$ & $0.2 \mathrm{~m}$ & 1 & $100 * 1 \mathrm{u}$ \\
\hline Latent heat $\left(h_{f g}\right)$ & $3.869 \times 10^{5} \mathrm{~J} / \mathrm{kg}$ & $2.0705 \times 10^{12}$ & $5.7514 \times 10^{6} l^{2}{ }^{2} . \mathrm{ts}^{-2}$ \\
\hline Melting temperature $\left(T_{x=L}\right)$ & $933 \mathrm{~K}$ & 0.912 & 0.912 tu \\
\hline Subjected temperature $\left(T_{x=0}\right)$ & $1023 \mathrm{~K}$ & 1 & 1 tu \\
\hline Density $(\rho)$ & $2698.9 \mathrm{~kg} / \mathrm{m}^{3}$ & $1^{*}$ & $1 * \mathrm{mu}^{\prime} \mathrm{lu}^{-3}$ \\
\hline Specific heat $\left(C_{p}\right)$ & $900 \mathrm{~J} / \mathrm{kg} . \mathrm{K}$ & $4.9272 \times 10^{12}$ & $1.3687 \times 10^{7} \mathrm{lu}^{2} . \mathrm{ts}^{-2} \cdot \mathrm{tu}^{-1}$ \\
\hline Thermal conductivity $(k)$ & $210 \mathrm{~W} / \mathrm{m} . \mathrm{K}$ & $4.9272 \times 10^{12}$ & $2.2811 \times 10^{6}$ mu.lu.ts ${ }^{-3} \cdot \mathrm{tu}^{-1}$ \\
\hline Thermal diffusivity $(\alpha)$ & $8.6455 \times 10^{-5} \mathrm{~m}^{2} / \mathrm{s}$ & $1 *$ & $0.1667 \mathrm{lu}^{2} \cdot \mathrm{ts}^{-1}$ \\
\hline
\end{tabular}

Table 6. Converting data for phase change problem. The values with * represent assumed values.
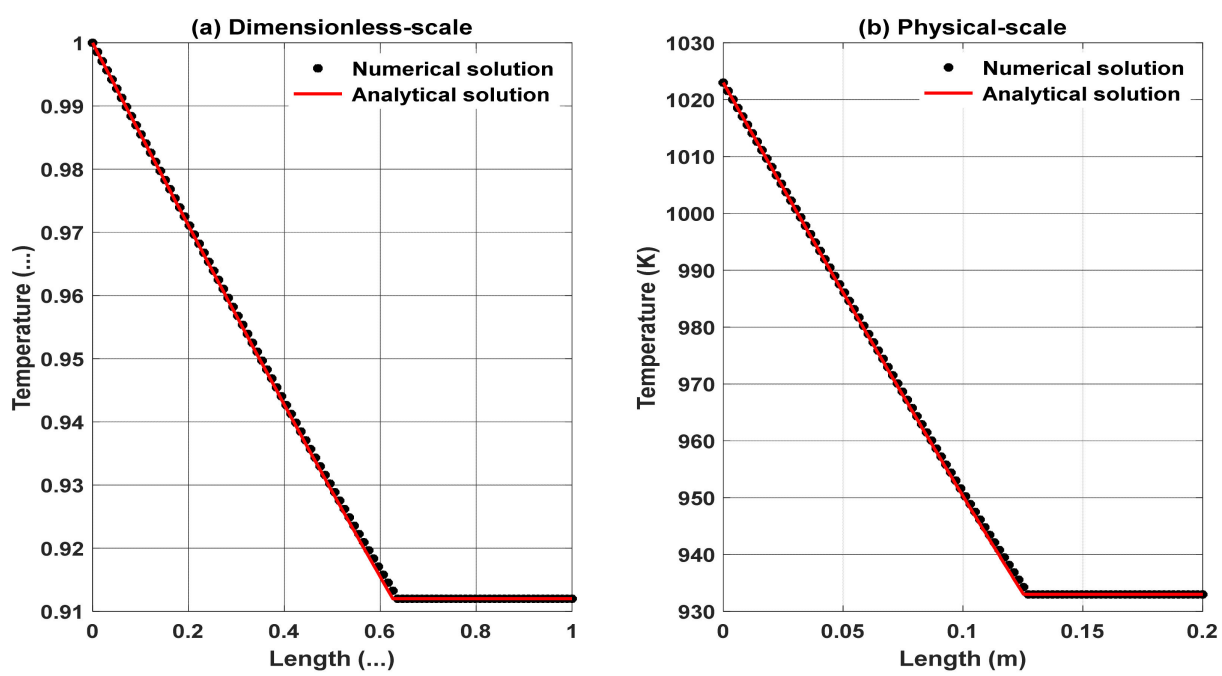

Figure 8. Temperature distribution along the slab for dimensionless time $t^{*}=1$ (i.e., for $4.6267 \times 10^{2} \mathrm{~s}$ ); (a) solution at the dimensionless-scale, and (b) solution at the physical-scale. The analytical solutions are achieved by using Equation (81)) at the dimensionless-scale and physical-scale. The numerical solution is achieved by using the LBM at the dimensionless-scale. 


\subsection{Two-Dimensional Examples}

The D2Q9 lattice model is used to solve the Navier-Stokes equations, while the D2Q5 for the energy equation. $f_{i}$ is a distribution function used for the D2Q9, while $h_{i}$ for the D2Q5. The models have the following equilibrium distribution function, lattice weights, lattice velocities, and speed of sound.

D2Q9:

$$
\begin{aligned}
& f_{i}^{e q}=w_{i} \rho\left[1+\frac{\boldsymbol{e}_{i} \cdot \boldsymbol{u}^{e q}}{C_{s}^{2}}+\frac{\left(\boldsymbol{e}_{i} \cdot \boldsymbol{u}^{e q}\right)^{2}}{2 C_{s}^{4}}-\frac{\left(\boldsymbol{u}^{e q}\right)^{2}}{2 C_{s}^{2}}\right], \\
& w_{i}=\left\{\begin{array}{cc}
4 / 9 & i=0 \\
1 / 9 & i=1,2,3,4, \\
1 / 36 & i=5,6,7,8
\end{array}\right. \\
& \boldsymbol{e}_{i}=\left[\begin{array}{ccccccccc}
0 & 1 & 0 & -1 & 0 & 1 & -1 & -1 & 1 \\
0 & 0 & 1 & 0 & -1 & 1 & 1 & -1 & -1
\end{array}\right] \text {, }
\end{aligned}
$$

and

$$
C_{s}^{2}=\frac{1}{3} .
$$

D2Q5:

$$
\begin{gathered}
h_{i}^{e q}=w_{i} \rho\left[1+\frac{\boldsymbol{e}_{i} \cdot \boldsymbol{u}^{e q}}{C_{s}^{2}}\right], \\
w_{i}=\left\{\begin{array}{cc}
2 / 6 & i=0 \\
1 / 6 & i=1,2,3,4
\end{array},\right. \\
\boldsymbol{e}_{i}=c\left[\begin{array}{lllcc}
0 & 1 & 0 & -1 & 0 \\
0 & 0 & 1 & 0 & -1
\end{array}\right],
\end{gathered}
$$

and

$$
C_{s}^{2}=\frac{1}{3} .
$$

\subsubsection{D Flow in a Channel}

This example is characterized by the stability and accuracy criteria. Air flows in a channel with $8 \mathrm{~mm} / \mathrm{s}$ of an average velocity $(V)$ at the inlet. The channel has $1.5 \mathrm{~m}$ of a length $(L)$ and $20 \mathrm{~cm}$ of gap $(H)$. The pressure drop $(\partial P / \partial x)$ inside the channel is $-4.54 \times 10^{-5} \mathrm{~Pa} / \mathrm{m}$. The density $(\rho)$ and dynamic viscosity $(\mu)$ of air are $1.2 \mathrm{~kg} / \mathrm{m}^{3}$ and $1.89 \times 10^{-5} \mathrm{~kg} / \mathrm{m} . \mathrm{s}$, respectively. The analytical solution of the velocity distribution at a full development regime is $[27,28]$ :

$$
u=\frac{H^{2}}{2 \mu}\left(\frac{\partial P}{\partial x}\right)\left[\left(\frac{y}{H}\right)^{2}-\left(\frac{y}{H}\right)\right] .
$$

The following data are used in the LBM simulation:

$$
\begin{gathered}
\tau_{f}=0.7, \\
\rho \boldsymbol{u}^{e q}=\sum f_{i} e_{i},
\end{gathered}
$$

and

$$
S_{i}=0 .
$$

The LBM simulation of flow in a channel is performed using the tabled data, Table 7. Figure 9 demonstrates the fully developed velocity profile. As shown, good agreements between the LBM and analytical solutions are achieved. To show the effect of the lattice Mach number $\left(M a_{(L S)}\right)$ on the accuracy of the LBM, Figure 10 is plotted. As seen in Figure 10, as the $M a_{(L S)}$ increases, the error between the LBM and analytical results 
increases too. The error is due to the pseudo-incompressibility of the LBM. Based on the numerical experimentations, the stability limit of the LBM is at $M a_{(L S)}$ equals 0.49 , where the scheme becomes unstable beyond this number.

Table 7. Converting data for flow in a channel problem. The values with * represent assumed values.

\begin{tabular}{|c|c|c|c|}
\hline \multicolumn{4}{|c|}{ Reference Variables in Physical-Scale and Lattice-Scale } \\
\hline Reference Variables & \multicolumn{2}{|c|}{ Physical-Scale } & Lattice-Scale \\
\hline$m_{0}$ & \multicolumn{2}{|c|}{$9.6 \times 10^{-3} \mathrm{~kg}$} & $2.49 \times 10^{6} \mathrm{mu}$ \\
\hline$x_{0}$ & \multicolumn{2}{|c|}{$0.2 \mathrm{~m}$} & $135.45 \mathrm{lu}$ \\
\hline$t_{0}$ & \multicolumn{2}{|c|}{$25 \mathrm{~s}$} & 2708.99 ts \\
\hline \multicolumn{4}{|c|}{ Converting properties between scales } \\
\hline Property & Physical-scale & Dimensionless-scale & Lattice-scale \\
\hline $\operatorname{Gap}(H)$ & $0.2 \mathrm{~m}$ & 1 & $135.45 \mathrm{lu}$ \\
\hline Length $(L)$ & $1.5 \mathrm{~m}$ & 7.5 & $1015.87 \mathrm{lu}$ \\
\hline Velocity $(V)$ & $0.008 \mathrm{~m} / \mathrm{s}$ & $1 *$ & $0.05 *$ lu.ts ${ }^{-1}$ \\
\hline Pressure drop $(\partial P / \partial x)$ & $-4.54 \times 10^{-5} \mathrm{~Pa} / \mathrm{m}$ & -0.1181 & $-2.18 \times 10^{-6} \mathrm{mu} \cdot \mathrm{lu}^{-2} \cdot \mathrm{ts}^{-2}$ \\
\hline Density $(\rho)$ & $1.2 \mathrm{~kg} / \mathrm{m}^{3}$ & $1 *$ & $1 * \mathrm{mu} \mathrm{lu} \mathrm{u}^{-3}$ \\
\hline Dynamic viscosity $(\mu)$ & $1.89 \times 10^{-5} \mathrm{~kg} / \mathrm{m} . \mathrm{s}$ & $9.844 \times 10^{-3}$ & 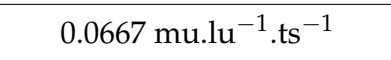 \\
\hline Kinematic viscosity $(v)$ & $1.58 \times 10^{-5} \mathrm{~m}^{2} / \mathrm{s}$ & $9.844 \times 10^{-3}$ & $0.0667 \mathrm{lu}^{2} . \mathrm{ts}^{-1}$ \\
\hline
\end{tabular}
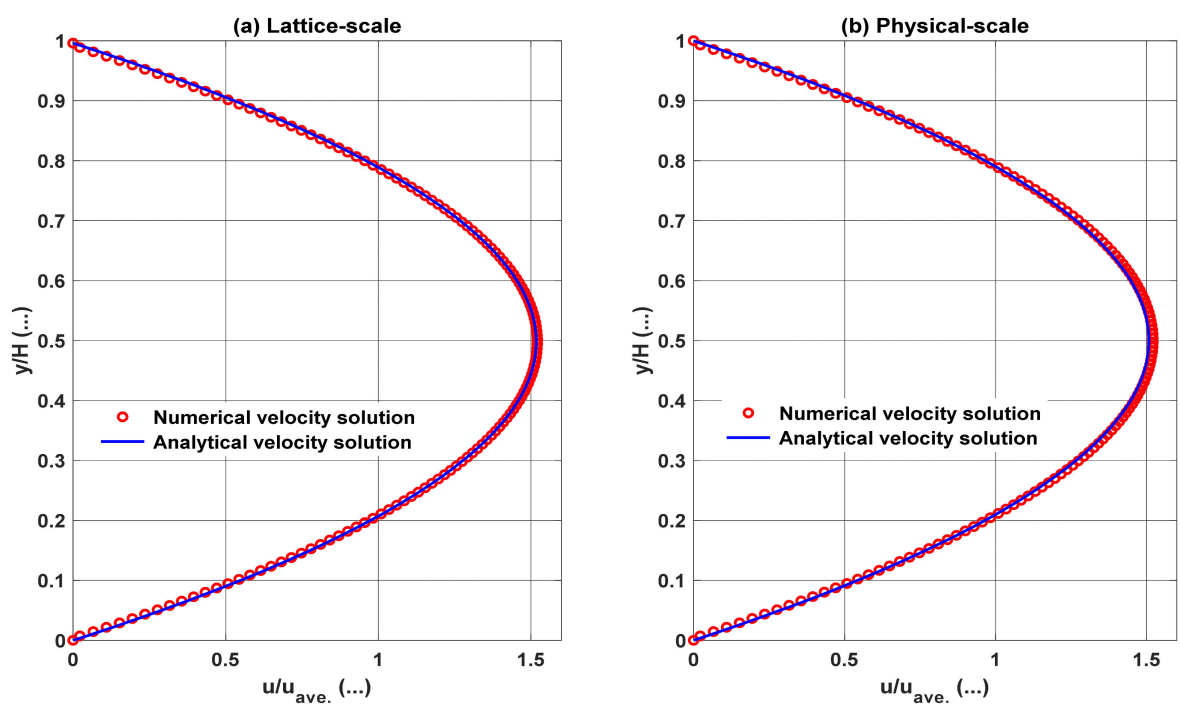

Figure 9. Fully developed velocity profile; (a) solutions at the lattice-scale, and (b) solutions at the physical-scale.

\subsubsection{D Differentially Heated Square Cavity}

A square cavity filled with air at a room temperature ( $298.15 \mathrm{~K})$ is driven by natural convection. The cavity has $0.02 \mathrm{~m}$ in length. The air properties at room temperature are summarized in Table 8. The west wall of the cavity is heated to a temperature of $1549.27 \mathrm{~K}$. This value is selected to ensure $\mathrm{Ra}=10^{6}$ for comparison with the benchmark solution. The east wall is kept at room temperature. The other boundaries are adiabatic. In such problems, the buoyancy force generated by the heated wall drives the flow inside the cavity $[4,11,29-31]$ :

$$
\boldsymbol{F}=\rho \boldsymbol{g} \beta\left(T-T_{\text {ref }}\right)
$$


where $T_{r e f}$ is the reference temperature, which equals the average temperature between west and east walls.

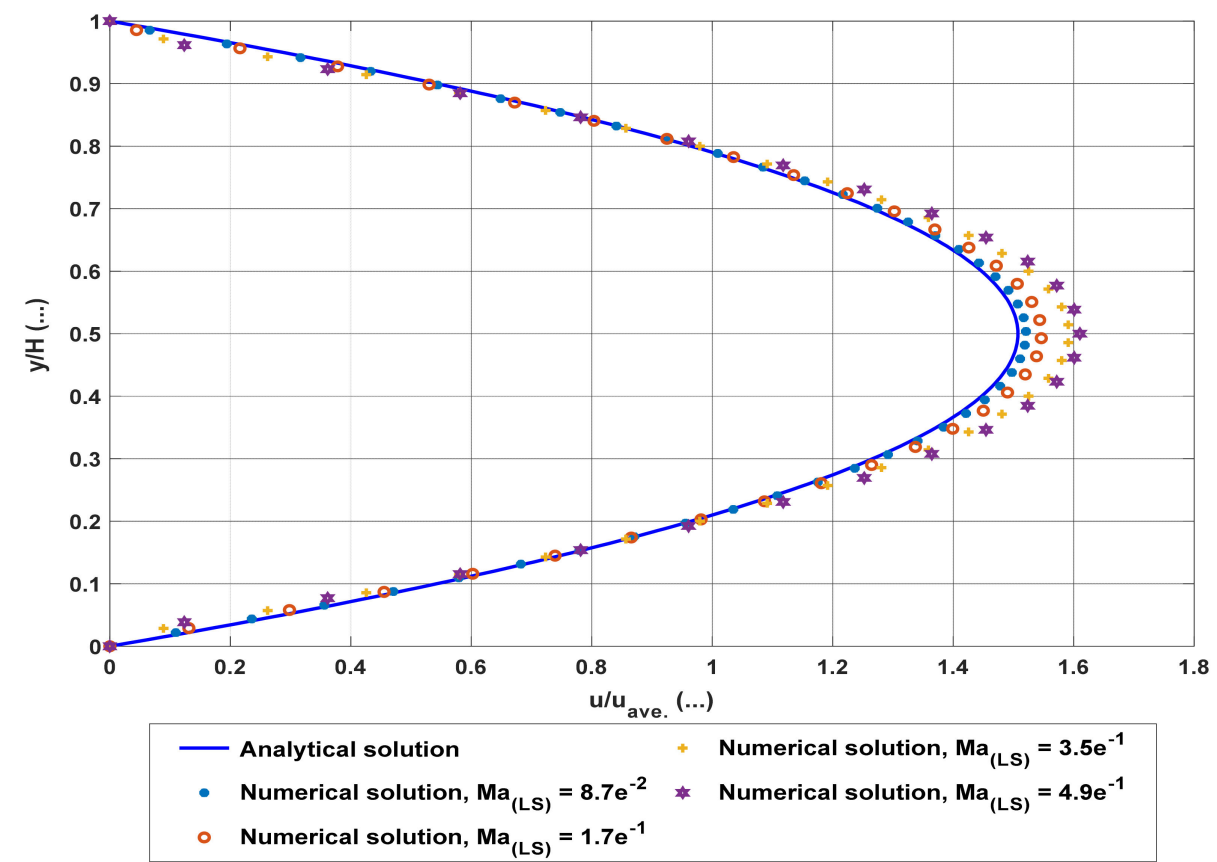

Figure 10. Effect of the lattice Mach number $\left(M a_{(L S)}\right)$ on the accuracy of the LBM.

Table 8. Converting data for the differentially heated square cavity example. The values with * represent assumed values.

\begin{tabular}{|c|c|c|c|}
\hline \multicolumn{4}{|c|}{ Reference Variables in Physical-Scale and Lattice-Scale } \\
\hline Reference Variables & \multicolumn{2}{|c|}{ Physical-Scale } & Lattice-Scale \\
\hline$m_{0}$ & \multicolumn{2}{|c|}{$9.6 \times 10^{-6} \mathrm{~kg}$} & $6.4 \times 10^{7} \mathrm{mu}$ \\
\hline$x_{0}$ & \multicolumn{2}{|c|}{$0.02 \mathrm{~m}$} & $400 \mathrm{lu}$ \\
\hline$t_{0}$ & \multicolumn{2}{|c|}{$18.6916 \mathrm{~s}$} & $1.7495 \times 10^{6}$ ts \\
\hline$\Theta_{o}$ & \multicolumn{2}{|c|}{$1549.27 \mathrm{~K}$} & $1 * \mathrm{tu}$ \\
\hline \multicolumn{4}{|c|}{ Converting properties between scales } \\
\hline Property & Physical-scale & Dimensionless-scale & Lattice-scale \\
\hline Length $(L)$ & $0.02 \mathrm{~m}$ & 1 & $400 * 1 \mathrm{u}$ \\
\hline Room temperature $\left(T_{\text {room }}\right)$ & $298.15 \mathrm{~K}$ & 0.1924 & $0.1924 \mathrm{tu}$ \\
\hline West wall temperature $\left(T_{\text {west }}\right)$ & $1549.27 \mathrm{~K}$ & 1 & $1 \mathrm{tu}$ \\
\hline Density $(\rho)$ & $1.2 \mathrm{~kg} / \mathrm{m}^{3}$ & $1 *$ & $1 * \mathrm{mu} .1 u^{-3}$ \\
\hline viscosity $(v)$ & $1.56 \times 10^{-5} \mathrm{~m}^{2} / \mathrm{s}$ & $7.2897 \times 10^{-1}$ & $6.6667 \times 10^{-2} \mathrm{lu}^{2} \cdot \mathrm{ts}^{-1}$ \\
\hline Thermal diffusivity $(\alpha)$ & $2.14 \times 10^{-5} \mathrm{~m}^{2} / \mathrm{s}$ & $1 *$ & $9.1453 \times 10^{-2} \mathrm{lu}^{2} \cdot \mathrm{ts}^{-1}$ \\
\hline Coefficient of expansion $(\beta)$ & $3.4 \times 10^{-3} \mathrm{~K}^{-1}$ & 5.2675 & $5.2675 \mathrm{tu}^{-1}$ \\
\hline Gravity $(g)$ & $9.81 \mathrm{~m} / \mathrm{s}^{2}$ & $1.7137 \times 10^{5}$ & $2.2395 \times 10^{-5}$ lu.ts $^{-2}$ \\
\hline Magnitude velocity $(u)$ & $0.9136 \mathrm{~m} / \mathrm{s}$ & 853.7985 & 0.1952 lu.ts ${ }^{-1}$ \\
\hline
\end{tabular}

In the LBM simulation, the following relaxation times related to momentum and temperature equations are applied:

$$
\tau_{f}=0.7
$$


and

$$
\tau_{e}=0.7744
$$

Luo's $[32,33]$ forcing scheme is used in the example, which has:

$$
S_{i}=\frac{w_{i}}{C_{S}^{2}} \boldsymbol{e}_{i} \cdot \boldsymbol{F},
$$

and

$$
\rho \boldsymbol{u}^{e q}=\sum f_{i} e_{i} .
$$

The obtained LBM results are compared with those of Quere and De Roquefortt [34]. Table 9 shows a comparison between the LBM and Quere and De Roquefortt [34] results. In the table, $X_{\max }$ and $Y_{\max }$ are the locations of maximum $U_{\max }$ and $V_{\max }$, respectively. The average Nusselt number $\left(N u_{\text {ave }}\right)$ is estimated as:

$$
N u_{\text {ave }}=\frac{1}{\left(T_{\text {west }}-T_{\text {east }}\right)} \sum_{k=1}^{k=N} \frac{3 T_{w, k}-4 T_{w+1, k}+T_{w+2, k}}{2 \Delta x},
$$

where the subscripts $w, w+1$, and $w+2$ represent the lattice of the heated wall, the lattice adjacent to the heated wall, and the lattice after adjacent to the heated wall in the $\mathrm{x}$-direction, respectively. Table 9 shows a good agreement between the results of the current study and Quere and De Roquefortt.

Table 9. Dimensionless results for $R a=10^{6}$.

\begin{tabular}{cccccc}
\hline & $\mathbf{N} u_{\text {ave }}$ & $\boldsymbol{U}_{\max }$ & $\boldsymbol{Y}_{\max }$ & $\boldsymbol{V}_{\max }$ & $\boldsymbol{X}_{\max }$ \\
\hline Ref. [33] & 8.814 & 64.81 & 0.850 & 220.57 & 0.0378 \\
Present study & 8.6915 & 63.9960 & 0.8529 & 216.8242 & 0.0399 \\
\hline
\end{tabular}

\subsubsection{D Stationary Droplet}

In the following example, we considered a stationary droplet placed at the center of the vapor phase domain. Such a problem is usually used to evaluate the stability and accuracy of the multiphase model, as well as to estimate the surface tension using Laplace's law. In the current example, the surface tensions of water at different temperatures are estimated. Therefore, the inter-particle force between liquid and vapor phases can be presented as [35]:

$$
\boldsymbol{F}_{\text {int }}(\boldsymbol{x}, t)=-G \psi(\boldsymbol{x}, t) \sum_{i} \omega_{i} \psi\left(\boldsymbol{x}+\boldsymbol{e}_{i} \Delta t, t\right) \boldsymbol{e}_{i},
$$

where $G$ is a parameter that controls the strength of the inter-particle force and $\psi$ is the mean-field potential function. Yuan and Schaefer [36] modified the SC [6,7] model and defined $\psi$ as:

$$
\psi=\sqrt{\frac{2\left\{P_{\mathrm{EoS}}-C_{s}^{2} \rho\right\}}{C_{S}^{2} G}},
$$

where $P_{\text {EoS }}$ is the EoS pressure. The R-K EoS is used in the present example. The parameters $a$ and $b$ of the R-K EoS can be defined as [37]:

$$
a=1.282 \frac{R T_{c} \sqrt{T_{c}}}{\rho_{c}},
$$

and

$$
b=\frac{0.2597}{\rho_{c}},
$$

where

$$
R T_{c}=0.27847,
$$


and

$$
\rho_{c}=\ln (2) \rho_{o},
$$

where $\rho_{o}$ is an arbitrary constant. The values of $R$ and $\rho_{o}$ are set to be 1 and 20 for water, respectively.

Table 10 shows the scaling parameters that are used to map the data from the latticescale to the physical-scale. The LBM predictions are compared with Jain et al. [38] LBM simulations and with the DDBST GmbH database [39], as shown in Figure 11. It is clear that there is a good agreement between the current predictions with published results.

Table 10. Scaling units between SI and lattice systems.

\begin{tabular}{ccc}
\hline Primary Dimension & Physical-Scale & Lattice-Scale \\
\hline Mass & $1 \mathrm{~kg} / \mathrm{kg}$ & $2.0972 \times 10^{3} \mathrm{mu} / \mathrm{kg}$ \\
\hline Length & $1 \mathrm{~m} / \mathrm{m}$ & $128 \mathrm{lu} / \mathrm{m}$ \\
\hline Time & $1 \mathrm{~s} / \mathrm{s}$ & $4.4184 \times 10^{-3} \mathrm{ts} / \mathrm{s}$ \\
\hline Temperature & $1 \mathrm{~K} / \mathrm{K}$ & $4.3038 \times 10^{-4} \mathrm{tu} / \mathrm{K}$ \\
\hline
\end{tabular}

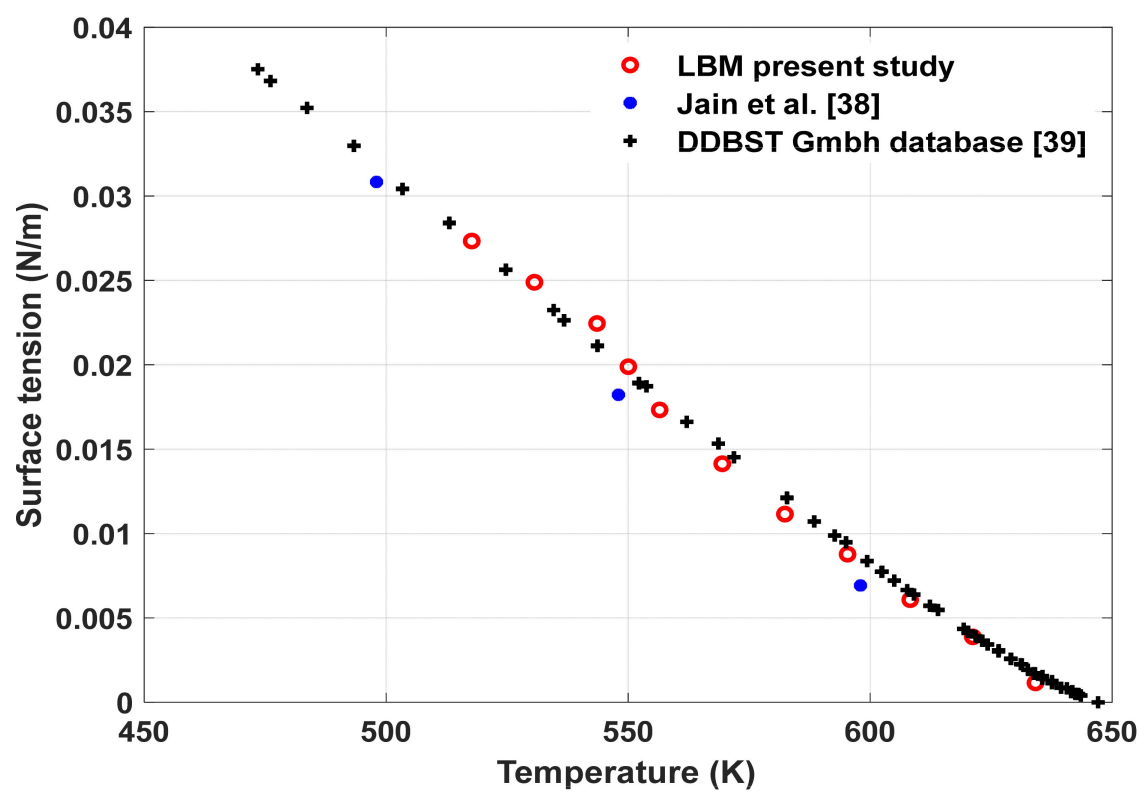

Figure 11. Comparison between the current work and previously published works for the surface tension of water as a function of temperature.

\section{Conclusions}

The article deals with converting units of the quantities from/to the physical-space to/from the lattice-space. A novel dimensional analysis method is proposed. The proposed approach is characterized by high flexibility and simplicity in controlling the stability and accuracy of LBM. The accuracy of LBM is strongly dependent on the lattice Mach number $\left(M a_{(L S)}\right)$, while its stability depends on the relaxation time. Increasing the lattice Mach number reduces the accuracy. Six benchmark examples are used to test the ability of the approach. The stability and accuracy of LBM are also evaluated. In conclusion, the proposed approach is a powerful and reliable tool to transform properties' units from/to the physical-scale to/from the lattice-scale.

Author Contributions: Conceptualization, S.S.B. and S.A.B.; methodology, S.S.B. and S.A.B.; software, S.S.B. and S.A.B.; validation, S.A.B.; formal analysis, S.S.B.; investigation, S.S.B., S.A.B. and A.A.M.; resources, A.A.M.; data curation, S.S.B. and S.A.B.; writing-original draft preparation, S.S.B.; writing—review and editing, S.A.B. and A.A.M.; visualization, S.S.B., S.A.B. and A.A.M.; 
supervision, A.A.M.; project administration, A.A.M.; funding acquisition, A.A.M. All authors have read and agreed to the published version of the manuscript.

Funding: This research received no external funding.

Institutional Review Board Statement: Not applicable.

Informed Consent Statement: Not applicable.

Acknowledgments: The authors acknowledge the National Science Engineering Research Council of Canada (NSERC) for their financial support.

Conflicts of Interest: The authors declare no conflict of interest.

\section{Appendix A}

Table A1 shows the conversion factors to go from the physical-scale to the dimensionlessscale and from the dimensionless-scale to the lattice-scale. To go in the other direction (i.e., from the dimensionless-scale to the physical-scale or from the lattice-scale to the dimensionlessscale) the relations in Table A1 need to be inverted (i.e., raised to power -1). For example, the conversion factor to scale velocity from the physical-scale to the dimensionless-scale can be obtained from Table A1 as:

$$
u^{*}=u_{(P S)} \frac{t_{o(P S)}}{x_{o(P S)}} \text {. }
$$

Then, the conversion factor to scale this velocity to the Lattice-scale $\left(\frac{x_{o(L S)}}{t_{o(L S)}}\right)$ is achieved from Table A1 as:

$$
u_{(L S)}=u^{*} \frac{x_{o(L S)}}{t_{o(L S)}}=u_{(P S)} \frac{t_{o(P S)}}{x_{o(P S)}} \frac{x_{o(L S)}}{t_{o(L S)}} .
$$

To go back from the lattice-scale to the dimensionless-scale, the conversion factor is inverted $\left(\left[\frac{x_{o(L S)}}{t_{o(L S)}}\right]^{-1}\right)$ :

$$
u^{*}=u_{(L S)} \frac{t_{o(L S)}}{x_{o(L S)}}
$$

Likewise, this velocity can be scaled to the physical-scale as:

$$
u_{(P S)}=u^{*} \frac{x_{o(P S)}}{t_{o(P S)}}=u_{(L S)} \frac{t_{o(L S)}}{x_{o(L S)}} \frac{x_{o(P S)}}{t_{o(P S)}},
$$

where the conversion factor is inverted $\left(\left[\frac{t_{o(P S)}}{x_{o(P S)}}\right]^{-1}\right)$.

The same procedure can be applied to scale the unit of any variable from/to the physical-scale to/from the lattice-scale using the listed data in Table A1.

Table A1. Factors to transform some selected quantities from/to the physical-scale to/from the dimensionless-scale to/from the lattice-scale.

\begin{tabular}{ccccc}
\hline Quantity & SI Unit & Dimensions & $\begin{array}{c}\text { Converting Factor from } \\
\text { Physical-Scale to } \\
\text { Dimensionless-Scale }\end{array}$ & $\begin{array}{c}\text { Converting Factor from } \\
\text { Dimensionless-Scale to } \\
\text { Lattice-Scale }\end{array}$ \\
\hline Length $(L)$ & $\mathrm{m}$ & $L$ & $\frac{1}{x_{o(P S)}}$ & $x_{o(L S)}$ \\
\hline Mass $(m)$ & $\mathrm{kg}$ & $M$ & $\frac{1}{m_{o(P S)}}$ & $m_{o(L S)}$ \\
\hline Temperature $(T)$ & $\mathrm{K}$ & $\Theta$ & $\frac{1}{\Theta_{o(P S)}}$ & $\Theta_{o(L S)}$ \\
\hline Time $(t)$ & $\mathrm{s}$ & $t$ & $\frac{1}{t_{o(P S)}}$ & $t_{o(L S)}$ \\
\hline Acceleration $(a, g)$ & $\mathrm{m} / \mathrm{s}^{2}$ & $L t^{-2}$ & $\frac{x_{o(L S)}}{t_{o(P S)}^{2}}$ & \\
\hline
\end{tabular}


Table A1. Cont.

\begin{tabular}{|c|c|c|c|c|}
\hline Quantity & SI Unit & Dimensions & $\begin{array}{l}\text { Converting Factor from } \\
\text { Physical-Scale to } \\
\text { Dimensionless-Scale }\end{array}$ & $\begin{array}{c}\text { Converting Factor from } \\
\text { Dimensionless-Scale to } \\
\text { Lattice-Scale }\end{array}$ \\
\hline Area $(A)$ & $\mathrm{m}^{2}$ & $L^{2}$ & $\frac{1}{x_{o(P S)}^{2}}$ & $x_{o(L S)}^{2}$ \\
\hline Angular velocity $(\omega, \Omega)$ & $\mathrm{rad} / \mathrm{s}$ & $t^{-1}$ & $t_{o(P S)}$ & $\frac{1}{t_{o(L S)}}$ \\
\hline Dynamic viscosity $(\mu)$ & Pa.s & $M L^{-1} t^{-1}$ & $\frac{t_{o(P S)} x_{o(P S)}}{m_{o(P S)}}$ & $\frac{m_{o(L S)}}{t_{o(L S)} x_{o(L S)}}$ \\
\hline Density $(\rho)$ & $\mathrm{kg} / \mathrm{m}^{3}$ & $M L^{-3}$ & $\frac{x_{o(P S)}^{3}}{m_{o(P S)}}$ & $\frac{m_{o(L S)}}{x_{o(L S)}^{3}}$ \\
\hline Kinematic viscosity $(v)$ & $\mathrm{m}^{2} / \mathrm{s}$ & $L^{2} t^{-1}$ & $\frac{t_{o(P S)}}{x_{o(P S)}^{2}}$ & $\frac{x_{o(L S)}^{2}}{t_{o(L S)}}$ \\
\hline Energy, work $(E, W)$ & $\mathrm{J}$ & $M L^{2} t^{-2}$ & $\frac{t_{o(P S)}^{2}}{m_{o(P S)} x_{o(P S)}^{2}}$ & $\frac{m_{o(L S)} x_{o(L S)}^{2}}{t_{o(L S)}^{2}}$ \\
\hline Energy per unit volume $\left(E^{\prime \prime \prime}\right)$ & $\mathrm{J} / \mathrm{m}^{3}$ & $M L^{-1} t^{-2}$ & $\frac{x_{o(P S)} t_{o(P S)}^{2}}{m_{o(P S)}}$ & $\frac{m_{o(L S)}}{x_{o(L S)} t_{o(L S)}^{2}}$ \\
\hline Expansion coefficient $(\beta)$ & $\mathrm{K}^{-1}$ & $\Theta^{-1}$ & $\Theta_{o(P S)}$ & $\frac{1}{\Theta_{o(L S)}}$ \\
\hline Force $(F)$ & $\mathrm{N}$ & $M L t^{-2}$ & $\frac{t_{o(P S)}^{2}}{x_{o(P S)} m_{o(P S)}}$ & $\frac{x_{o(L S)} m_{o(L S)}}{t_{o(L S)}^{2}}$ \\
\hline Force per unit volume $\left(F^{\prime \prime \prime}\right)$ & $\mathrm{N} / \mathrm{m}^{3}$ & $M L^{-2} t^{-2}$ & $\frac{t_{o(P S)}^{2} x_{o(P S)}^{2}}{m_{o(P S)}}$ & $\frac{m_{o(L S)}}{t_{o(L S)}^{2} x_{o(L S)}^{2}}$ \\
\hline Heat flux $\left(q^{\prime \prime}\right)$ & $\mathrm{W} / \mathrm{m}^{2}$ & $M t^{-3}$ & $\frac{t_{o(P S)}^{3}}{m_{o(P S)}}$ & $\frac{m_{o(L S)}}{t_{o(L S)}^{3}}$ \\
\hline Heat transfer coefficient $(h)$ & $\mathrm{W} / \mathrm{m}^{2} \cdot \mathrm{K}$ & $M t^{-3} \Theta^{-1}$ & $\frac{t_{o(P S)}^{3} \Theta_{o(P S)}}{m_{o(P S)}}$ & $\frac{m_{o(L S)}}{t_{o(L S)}^{3} \Theta_{o(L S)}}$ \\
\hline Mass flow $(\dot{m})$ & $\mathrm{kg} / \mathrm{s}$ & $M t^{-1}$ & $\frac{t_{o(P S)}}{m_{o(P S)}}$ & $\frac{m_{o(L S)}}{t_{o(L S)}}$ \\
\hline Moment, torque $(M)$ & N.m & $M L^{2} t^{-2}$ & $\frac{t_{o(P S)}^{2}}{x_{o(P S)}^{2} m_{o(P S)}}$ & $\frac{x_{o(L S)}^{2} m_{o(L S)}}{t_{o(L S)}^{2}}$ \\
\hline Pressure, stress $(p, \tau)$ & $\mathrm{Pa}$ & $M L^{-1} t^{-2}$ & $\frac{x_{o(P S)} t_{o(P S)}^{2}}{m_{o(P S)}}$ & $\frac{m_{o(L S)}}{x_{o(L S)} t_{o(L S)}^{2}}$ \\
\hline Power $(P)$ & W & $M L^{2} t^{-3}$ & $\frac{t_{o(P S)}^{3}}{x_{o(P S)}^{2} m_{o(P S)}}$ & $\frac{x_{o(L S)}^{2} m_{o(L S)}}{t_{o(L S)}^{3}}$ \\
\hline Specific enthalpy $(h)$ & $\mathrm{J} / \mathrm{kg}$ & $L^{2} t^{-2}$ & $\frac{t_{o(P S)}^{2}}{x_{o(P S)}^{2}}$ & $\frac{x_{o(L S)}^{2}}{t_{o(L S)}^{2}}$ \\
\hline Specific entropy (s) & $\mathrm{J} / \mathrm{kg} . \mathrm{K}$ & $L^{2} t^{-2} \Theta^{-1}$ & $\frac{t_{o(P S)}^{2} \Theta_{o(P S)}}{x_{o(P S)}^{2}}$ & $\frac{x_{o(L S)}^{2}}{t_{o(L S)}^{2} \Theta_{o(L S)}}$ \\
\hline $\begin{array}{l}\text { Specific heat capacity } \\
\qquad\left(C_{p}, C_{v}\right)\end{array}$ & $\mathrm{J} / \mathrm{kg} . \mathrm{K}$ & $L^{2} t^{-2} \Theta^{-1}$ & $\frac{t_{o(P S)}^{2} \Theta_{o(P S)}}{x_{o(P S)}^{2}}$ & $\frac{x_{o(L S)}^{2}}{t_{o(L S)}^{2} \Theta_{o(L S)}}$ \\
\hline Specific weight $(\gamma)$ & $\mathrm{N} / \mathrm{m}^{3}$ & $M L^{-2} t^{-2}$ & $\frac{t_{o(P S)}^{2} x_{o(P S)}^{2}}{m_{o(P S)}}$ & $\frac{m_{o(L S)}}{t_{o(L S)}^{2} x_{o(L S)}^{2}}$ \\
\hline Speed of sound $\left(a, C_{S}\right)$ & $\mathrm{m} / \mathrm{s}$ & $L t^{-1}$ & $\frac{t_{o(P S)}}{x_{o(P S)}}$ & $\frac{x_{o(L S)}}{t_{o(L S)}}$ \\
\hline Strain rate $(\dot{\varepsilon})$ & $1 / \mathrm{s}$ & $t^{-1}$ & $t_{o(P S)}$ & $\frac{1}{t_{o(L S)}}$ \\
\hline Surface tension $(\sigma)$ & $\mathrm{N} / \mathrm{m}$ & $M t^{-2}$ & $\frac{t_{o(P S)}^{2}}{m_{o(P S)}}$ & $\frac{m_{o(L S)}}{t_{o(L S)}^{2}}$ \\
\hline Thermal conductivity $(k)$ & $\mathrm{W} / \mathrm{m} . \mathrm{K}$ & $M L t^{-3} \Theta^{-1}$ & $\frac{t_{o(P S)}^{3} \Theta_{o(P S)}}{x_{o(P S)} m_{o(P S)}}$ & $\frac{x_{o(L S)} m_{o(L S)}}{t_{o(L S)}^{3} \Theta_{o(L S)}}$ \\
\hline Velocity $(v)$ & $\mathrm{m} / \mathrm{s}$ & $L t^{-1}$ & $\frac{t_{o(P S)}}{x_{o(P S)}}$ & $\frac{x_{o(L S)}}{t_{o(L S)}}$ \\
\hline Volume $(V)$ & $\mathrm{m}^{3}$ & $L^{3}$ & $\frac{1}{x_{o(P S)}^{3}}$ & $x_{o(L S)}^{3}$ \\
\hline Volume flow $(\dot{V}, Q)$ & $\mathrm{m}^{3} / \mathrm{s}$ & $L^{3} t^{-1}$ & $\frac{t_{o(P S)}}{x_{o(P S)}^{3}}$ & $\frac{x_{o(L S)}^{3}}{t_{o(L S)}}$ \\
\hline
\end{tabular}




\section{References}

1. Li, Q.; Luo, K.H.; Kang, Q.; He, Y.; Chen, Q.; Liu, Q. Lattice Boltzmann methods for multiphase flow and phase-change heat transfer. Prog. Energy Combust. Sci. 2016, 52, 62-105. [CrossRef]

2. Succi, S. The Lattice Boltzmann Equation: For Fluid Dynamics and Beyond; Oxford University Press: Oxford, UK, 2001.

3. Chen, S.; Doolen, G.D. Lattice Boltzmann method for fluid flows. Annu. Rev. Fluid Mech. 1998, 30, 329-364. [CrossRef]

4. Mohamad, A.A. Lattice Boltzmann Method: Fundamentals and Engineering Applications with Computer Codes, 2nd ed.; Springer: Berlin/Heidelberg, Germany, 2019.

5. Huang, H.; Sukop, M.; Lu, X. Multiphase Lattice Boltzmann Methods: Theory and Application; John Wiley \& Sons: Hoboken, NJ, USA, 2015.

6. Shan, X.; Chen, H. Lattice Boltzmann model for simulating flows with multiple phases and components. Phys. Rev. E 1993, 47 , 1815. [CrossRef] [PubMed]

7. Shan, X.; Chen, H. Simulation of nonideal gases and liquid-gas phase transitions by the lattice Boltzmann equation. Phys. Rev. E 1994, 49, 2941. [CrossRef]

8. Ahmed, T. Equations of State and PVT Analysis; Elsevier: Amsterdam, The Netherlands, 2013.

9. Smith, J.M. Introduction to Chemical Engineering Thermodynamics; ACS Publications: Washington, DC, USA, 1950.

10. Mobinipouya, M.R.; Barzegar, Z. Calculation of Density for Refrigerant Mixtures in Sub Critical Regions for Use in the Buildings. Int. J. Phys. Math. Sci. 2011, 5, 210-212.

11. Bawazeer, S.A.; Baakeem, S.S.; Mohamad, A.A. A Critical Review of Forcing Schemes in Lattice Boltzmann Method: 1993-2019. Arch. Comput. Methods Eng. 2021. [CrossRef]

12. Huang, H.; Lu, X.-Y. Relative permeabilities and coupling effects in steady-state gas-liquid flow in porous media: A lattice Boltzmann study. Phys. Fluids 2009, 21, 092104. [CrossRef]

13. Huang, H.; Wang, L.; Lu, X.-Y. Evaluation of three lattice Boltzmann models for multiphase flows in porous media. Comput. Math. Appl. 2011, 61, 3606-3617. [CrossRef]

14. Toghaniyan, A.; Zarringhalam, M.; Akbari, O.A.; Shabani, G.A.S.; Toghraie, D. Application of lattice Boltzmann method and spinodal decomposition phenomenon for simulating two-phase thermal flows. Phys. A Stat. Mech. Its Appl. 2018, 509, 673-689. [CrossRef]

15. Eshraghi, M.; Felicelli, S.D. An implicit lattice Boltzmann model for heat conduction with phase change. Int. J. Heat Mass Transf. 2012, 55, 2420-2428. [CrossRef]

16. Lin, Q.; Wang, S.; Ma, Z.; Wang, J.; Zhang, T. Lattice Boltzmann simulation of flow and heat transfer evolution inside encapsulated phase change materials due to natural convection melting. Chem. Eng. Sci. 2018, 189, 154-164. [CrossRef]

17. Huang, J.; Bao, C.; Jiang, Z.; Zhang, X. A general approach of unit conversion system in lattice Boltzmann method and applications for convective heat transfer in tube banks. Int. J. Heat Mass Transf. 2019, 135, 873-884. [CrossRef]

18. Bhatnagar, P.L.; Gross, E.P.; Krook, M. A model for collision processes in gases. I. Small amplitude processes in charged and neutral one-component systems. Phys. Rev. 1954, 94, 511. [CrossRef]

19. Wolf-Gladrow, D.A. Lattice-Gas Cellular Automata and Lattice Boltzmann Models: An Introduction; Springer: Berlin/Heidelberg, Germany, 2004.

20. Succi, S. The Lattice Boltzmann Equation: For Complex States of Flowing Matter; Oxford University Press: Oxford, UK, 2018.

21. Boltzmann, L. Lectures on Gas Theory; Courier Corporation: Chelmsford, MA, USA, 2012.

22. Bawazeer, S. Stability and Accuracy of Lattice Boltzmann Method. Master's Thesis, University of Calgary, Calgary, AB, Canada, 2013.

23. Versteeg, H.K.; Malalasekera, W. An Introduction to Computational Fluid Dynamics: The Finite Volume Method; Pearson Education: London, UK, 2007.

24. Shyy, W.; Udaykumar, H.; Rao, M.M.; Smith, R.W. Computational Fluid Dynamics with Moving Boundaries; CRC Press: Boca Raton, FL, USA, 1995.

25. Özısık, M.N. Heat Conduction; John Wiley \& Sons: Hoboken, NJ, USA, 1993.

26. Jiaung, W.-S.; Ho, J.-R.; Kuo, C.-P. Lattice Boltzmann method for the heat conduction problem with phase change. Numer. Heat Transf. Part B Fundam. 2001, 39, 167-187.

27. Pritchard, P.J.; Mitchell, J.W. Fox and McDonalds Introduction to Fluid Mechanics; John Wiley \& Sons: Hoboken, NJ, USA, 2016.

28. White, F.M. Fluid Mechanics; Tata McGraw-Hill Education: New York, NY, USA, 1979.

29. Mohamad, A.; Kuzmin, A. A critical evaluation of force term in lattice Boltzmann method, natural convection problem. Int. J. Heat Mass Transf. 2010, 53, 990-996. [CrossRef]

30. Mohamad, A.; El-Ganaoui, M.; Bennacer, R. Lattice Boltzmann simulation of natural convection in an open ended cavity. Int. J. Therm. Sci. 2009, 48, 1870-1875. [CrossRef]

31. Bawazeer, S.; Mohamad, A.; Oclon, P. Natural convection in a differentially heated enclosure filled with low Prandtl number fluids with modified lattice Boltzmann method. Int. J. Heat Mass Transf. 2019, 143, 118562. [CrossRef]

32. Luo, L.-S. Analytic solutions of linearized lattice Boltzmann equation for simple flows. J. Stat. Phys. 1997, 88, 913-926. [CrossRef]

33. Luo, L.-S. Lattice-Gas Automata and Lattice Boltzmann Equations for Two-Dimensional Hydrodynamics; School of Physics, Georgia Institute of Technology: Atlanta, GA, USA, 1993.

34. Le Quéré, P.; de Roquefortt, T.A. Computation of natural convection in two-dimensional cavities with Chebyshev polynomials. J. Comput. Phys. 1985, 57, 210-228. [CrossRef] 
35. Martys, N.S.; Chen, H. Simulation of multicomponent fluids in complex three-dimensional geometries by the lattice Boltzmann method. Phys. Rev. E 1996, 53, 743. [CrossRef]

36. Yuan, P.; Schaefer, L. Equations of state in a lattice Boltzmann model. Phys. Fluids 2006, 18, 042101. [CrossRef]

37. Baakeem, S.S.; Bawazeer, S.A.; Mohamad, A. Comparison and evaluation of Shan-Chen model and most commonly used equations of state in multiphase lattice Boltzmann method. Int. J. Multiph. Flow 2020, 128, 103290. [CrossRef]

38. Jain, P.K.; Tentner, A.; Uddin, R. A lattice Boltzmann framework to simulate boiling water reactor core hydrodynamics. Comput. Math. Appl. 2009, 58, 975-986. [CrossRef]

39. Database, D.G. Surface Tension Data. Available online: http://www.ddbst.com/en/EED/PCP/SFT_C174.php (accessed on 24 June 2021). 